\title{
Long non-coding RNA SLC2A1-AS1 induced by GLI3 promotes aerobic glycolysis and progression in esophageal squamous celi carcinoma by sponging miR-378a-3p enhance Glut1 expression
}

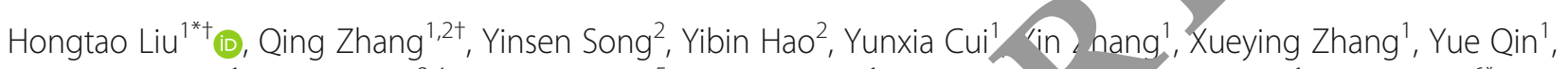
Guangzhao Zhu', Feng Wang ${ }^{3,4}$, Jinghan Dang ${ }^{5}$, Shanshan Ma', Yanting Zh, 'g 'Venna Guo', Shenglei Li ${ }^{6 *}$, Fangxia Guan ${ }^{1 *}$ and Tianli Fan ${ }^{7 *}$

\section{Abstract}

Background: Emerging evidence demonstrates that acRN, play pivotal roles in tumor energy metabolism; however, the detailed mechanisms of IncRNAs in ae re gulatı $n$ of tumor glycolysis remain largely unknown.

Methods: The expression of SLC2A1-AS1 was rinest, ted Dy TCGA, GEO dataset and qRT-PCR. The binding of GLI3 to SLC2A1-AS1 promoter was detected by $\angle \mathrm{c}$ ferase $\mathrm{k}$ porter Assay System and Ago2-RIP assay. FISH was performed to determine the localization o SLC 1-AS1 in ESCC cells. Double Luciferase Report assay was used to investigate the interaction of miR-379 a-3p with S! C2A1-AS1 and Glut1. Gain-of-function and Loss-of-function assay were performed to dissect the funct $n$ of SL/2A1-AS1/miR-378a-3p/Glut1 axis in ESCC progression in vitro and in vivo.

Results: We identified a novel ICrn C2A1-AS1 in ESCC. SLC2A1-AS1 was frequently overexpressed in ESCC tissues and cells, and its overexprossion as associated with TNM stage, lymph node metastasis and poor prognosis of ESCC patients. Importantly, 13 , hd SLC ZA1-AS1 formed a regulatory feedback loop in ESCC cells. SLC2A1-AS1 promoted cell growth in vitro ana VIVo, nigration and invasion, and suppressed apoptosis, leading to EMT progression and increased glyco, is in ESC Cells. SLC2A1-AS1 functioned as ceRNA for sponging miR-378a-3p, resulting in Glut1 overexpression in L CC celis. MiR-378a-3p inhibited cell proliferation and invasion as well as induced apoptosis, resulting 1 reduced o colysis, which was partly reversed by SLC2A1-AS1 or Glut1 overexpression in ESCC cells.

*Corrom_liuht1230@126.com; Islbljys@126.com;

guanfar.a@126.com; fantianlipp@163.com

${ }^{+}$Hongtao, $u$ and Qing Zhang contributed equally to this work.

${ }^{1}$ School of Life Sciences, Zhengzhou University, Zhengzhou 450001, Henan,

China

${ }^{6}$ Department of Pathology, the First Affiliated Hospital of Zhengzhou

University, 40 Daxue Road, Zhengzhou 450052, Henan, China

${ }^{7}$ Department of Pharmacology, School of Basic Medicine, Zhengzhou

University, 100 Kexue Road, Zhengzhou 450001, Henan, China

Full list of author information is available at the end of the article

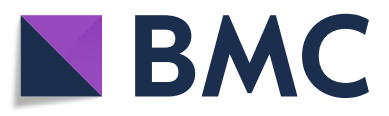

(c) The Author(s). 2021, corrected publication 2021. Open Access This article is licensed under a Creative Commons Attribution 4.0 International License, which permits use, sharing, adaptation, distribution and reproduction in any medium or format, as long as you give appropriate credit to the original author(s) and the source, provide a link to the Creative Commons licence, and indicate if changes were made. The images or other third party material in this article are included in the article's Creative Commons licence, unless indicated otherwise in a credit line to the material. If material is not included in the article's Creative Commons licence and your intended use is not permitted by statutory regulation or exceeds the permitted use, you will need to obtain permission directly from the copyright holder. To view a copy of this licence, visit http://creativecommons.org/ licenses/by/4.0/. The Creative Commons Public Domain Dedication waiver (http://creativecommons.org/publicdomain/zero/1. 0/) applies to the data made available in this article, unless otherwise stated in a credit line to the data. 
Conclusion: SLC2A1-AS1 plays important roles in ESCC development and progression by regulating glycolysis, and SLC2A1-AS1/miR-378a-3p/Glut1 regulatory axis may be a novel therapeutic target in terms of metabolic remodeling of ESCC patients.

Keywords: Esophageal squamous cell carcinoma, SLC2A1-AS1, miR-378a-3p, Glucose transporter 1, Glycolyşs

\section{Background}

Esophageal cancer (ESCA) is the seventh leading cause of cancer-related death in the world, which consists of two histological types, esophageal squamous cell carcinoma (ESCC) and esophageal adenocarcinoma (EAC) [1, 2]. In China, ESCC accounts for $90 \%$ of all ESCA types, and mainly distributes in Lin County and An Yang area, Henan province [3]. Although tremendous advances in diagnosis and treatment of ESCC patients, the 5-year survival rate for ESCC patients is quite poor [4, 5], which may be mainly due to diagnosis at an advanced stage, high recurrence and metastasis $[6,7]$. Therefore, it is extremely urgent for us to seek for novel prognostic markers and therapeutic targets for ESCC patients.

Tumor cells mainly obtain energy via a high rate or glycolysis for tumor survival, and this metabolic fer are is now considered as one of tumor hallmarks $\left[8, \mathrm{Qtt}_{\mathrm{J}}\right.$ Warburg has verified that tumor cells prefere atally take glucose as main energy source to pro a h lactate, even in the present of plentiful oxygen to sup th the increased metabolic rate [9], which is well known as "Warburg effect" or "aerobic glycolys. "10]. The metabolic characteristics is implicated in a su of the alteration of metabolic enzymes, suc 1 a ${ }^{1}$ 1ncose transporter 1 (Glut1, also named SLC2A1), hexokinase 2 (HK2), pyruvate kinase type $\mathrm{N} 2(\mathrm{KM} 2)$ and lactate dehydrogenase A (LDHA) 11 . . ably, many transcriptional factors inc fing $\mathrm{C}-\mathrm{L}_{\mathbf{+}}$, hypoxia inducible factor$1 \alpha$ (HIF-1 $\alpha)$, r. icle. factor kappa-B (NF-kB) promote tumor lact genesis $b$ regulating the expressions of metaboli. ${ }^{2}$ nnes $[17-19]$. Recent studies have demonstrated that of ing glycolysis-related pathways may be a $r$ vel herap utic target for a variety of tumors [2022], ICrr mill link many metabolic enzymes involved in glycolys Despite tremendous advances in tumor glycolysis, its regulatory mechanisms implicated its metabolic enzymes and transcriptional factors remain to be further identified.

Long non-coding RNAs (lncRNAs) as key regulatory elements of cell biological processes are longer than 200 nucleotides, without protein-coding potential, which is transcribed by RNA polymerase II [23]. LncRNAs are considered to be involved in many biological processes, including gene expression regulation, decoy for transcription factors, competing endogenous RNAs (ceRNAs) and scaffolding, etc. [24, 25]. Increasing reports have demonstrated that IncRNAs widely participate in cell growth, survival, differentiation ap tosis invasion and metastasis [26-29]. Recently IncRNAs ve been reported to be tightly associate with thinor glycolysis [30-32], and thus targeting hese $\mathrm{R}^{\mathrm{N}}$ As may be novel therapeutic strategies fo va ety of tumors. Recently, SLC2A1-AS1 as a $\mathrm{n}, \operatorname{lncRN}$, has been verified to promote tumor proifera $n$ by absorbing miR-508-5p in lung adenocr. $\mathrm{C}$ ma (LI AD) [33]. Besides, SLC2A1AS1 triggerea e expression of Glut1 expre ther resulting in $\mathrm{t}$. inhibition of glycolysis of hepatocellular ca ma (- $\mathrm{CCC}$ ) by competitively binding to transketol $s$, ar a signal transducer and activator of transcription 3 (STAT3) [34]. Our previous report revealed dit ential expression of lncRNAs in ESCA [35], and SLC2 1-AS1 was given our exclusive attention due to in - ame human chromosome 1 with Glut1, suggesting that it may be implicated in the regulation of glycolysis in ESCA. However, its roles and regulatory mechanisms involved in glycolysis remain elusive.

In the present study, we reported a novel lncRNA SLC2A1-AS1 in ESCC. We found that SLC2A1-AS1 was frequently overexpressed in ESCC tissues and cells, which might be due to the high expression of transcription factor GLI3, and its depletion suppressed ESCC cell growth in vitro and in vivo, migration and invasion as well as glycolysis, and induced cell apoptosis in ESCC cells. Mechanistically, SLC2A1-AS1 sponged miR-378a$3 p$ to enhance the Glut1 expression, which further triggered the increased glycolysis in ESCC. Taken altogether, our current data presented herein suggest that SLC2A1-AS1/miR-378a-3p/Glut1 regulatory axis plays an essential role in ESCC development and progression, and thus targeting the signal axis may be a novel therapeutic target for ESCC patients.

\section{Materials and methods}

\section{Tissue samples}

Sixty cases of ESCC samples and paired normal esophageal epithelial tissues were obtained during surgical resection at the First Affiliated Hospital of Zhengzhou University from 2010 to 2016. Tissue samples confirmed using H\&E staining by experienced pathologists were immediately frozen in liquid nitrogen after resection, which was further investigated by quantitative real-time PCR (qRT-PCR), Western blot and immunohistochemistry (IHC). Informed consent of all tissue samples was obtained from each patient and the utilization of tissue 
samples was approved by the Research and Ethics Committee of Zhengzhou University in this study. Meanwhile, the study methodologies completely conformed to the standards set by the Declaration of Helsinki.

\section{IHC assay}

IHC assay was performed according to previous reports [36]. In brief, tissue slides were fixed using formalin, embedded in paraffin and cut continuously for 4-6 $\mu \mathrm{m}$. After deparaffinization, rehydration and antigen retrieval using microwave heating in citrate buffer ( $\mathrm{pH}$ 6.0). Primary antibodies against Glut1 (Abcam, Cambridge, MA, USA) were incubated with tissue sections. After rinsing, the corresponding second antibody was added to tissue slides. Staining signals were developed using DAB reagent. The staining results were evaluated by two excellent pathologists.

\section{Public database assay}

Gene expression profiles from ESCA patients were downloaded from The Cancer Genome Atlas (TCGA) dataser using the GDC data portal (https://portal.gdc.cancer sow repository), which was analyzed according to our $\mathrm{p}$ riov publication [35]. GEO DataSet (GSE111011) w a app d to examine the SLC2A1-AS1, GLI3 and Glu 1 rpressio in 7 cases of ESCC patients and 7 cases of pairea ormal samples, and GSE43732 was utilized detect the miR378a-3p expression in 119 cases of FSC t/ssues and paired normal tissues. TCGA databas c. In StarBase (ENCORI) (The Encyclopedia or in. Interactomes) was used to investigate the SLC2A1- $S_{1}, C_{1} I 3, m i R-378 a-3 p$ and Glut1 expression in 16. cases of ESCA patients and 11 cases of normal samp 11011 was performed to analyze the expre ion of 8 colysis-related proteins in 7 cases of ESCC t ssuc and corresponding normal tissues.

\section{Cell lines, " alkul and transfection}

Hum ESCO KY E70 KYSZ30, KYSE180, KYSE450, KYSE510 and KYS ${ }_{\llcorner}, 0$ as well as normal esophageal epithelial cell Het-1A were obtained from the Chinese Academy of Sciences Cell Bank, which was maintained in RMPI 1640 medium supplemented with 10\% Fetal Bovine Serum (Gibco, Invitrogen, USA) in a humidified incubator harboring 5\% $\mathrm{CO}_{2}$. GLI3 siRNA \#1, 2 and 3, control siRNA (con-siRNA), SLC2A1-AS1 siRNA \#1 and 2, negative control (NC) (Supplementary Table 1), pcDNA3.1, pcDNA3.1-SLC2A1-AS1, pcDNA3.1-GLI3, pcDNA3.1Glut1 (Supplementary Table 2), NC-mimic, miR-378a3p mimic, NC-inhibitor, miR-378a-3p inhibitor (GenePharma Company, Shanghai, China), control siRNA and Glut1 siRNA (Santa Cruz company, USA) were transfected into EC9706, TE1 and KYSE180 cells by
Lipofectamine $^{\mathrm{Tm}} 2000$ (Invitrogen Life Technologies, Carslbad, CA, USA) according to manufacturer's instruction.

Cell counting Kit-8 (CCK-8) assay for cell prol; atir . CCK-8 kit was employed to evaluate cell prolit tio in ESCC cells. Briefly, various treatr int ESCO cells (EC9706, TE1 and KYSE180 cells) t a sity of $2 \times 10^{3}$ cells per well were seeded into $\%$-well plat 3 . At the indicated time points, CCK-8 (Be time B otech, Haimen, China) was added to corr. ona. ells according to manufacturer's protoco', and sorbance value at 450 $\mathrm{nm}$ was determined $\mathrm{A}$, nicropl ite reader (Thermo Scientific, Waltham, MA).

\section{Colony formatic}

Colony farmation operformed to access the cell proliferation a Itry Rrieily, ESCC cells $\left(1 \times 10^{3}\right)$ with different transfectio we added to 6 -well plate for $72 \mathrm{~h}$. Then, froch mediu 4 was used to replace the old medium to culture SCC cells for continuous 10 days. Finally, ESCC cells was xed using $4 \%$ of paraformaldehyde for $1 \mathrm{~h}$ and s. red with $0.1 \%$ crystal violet for $30 \mathrm{~min}$.

\section{EdU staining assay}

EdU staining assay was performed according to manufacturer's protocol. EC9706, TE1 and KYSE180 cells $\left(6 \times 10^{3}\right.$ cells/well $)$ were seeded into 96-well plate, and then were transfected with NC, SLC2A1-AS1 siRNA, pcDNA3.1 and pcDNA3.1-SLC2A1-AS1 according to manufacturer's protocol. Cells were labeled with EdU reagent in a final concentration of $50 \mu \mathrm{M}$ for $2 \mathrm{~h}$. Cells were rinsed using PBS buffer for $5 \mathrm{~min}$. Subsequently, cells was fixed in PBS buffer containing 4\% polyformaldehyde for $30 \mathrm{~min}$, and glycine $(2 \mathrm{mg} / \mathrm{ml})$ in a volume of $50 \mu \mathrm{l}$ was added to cells for $5 \mathrm{~min}$. Finally, TritonX-100 in a volume of $100 \mu \mathrm{l}$ was used for decolorization for 10 min. Regarding Apollo staining, a total of $100 \mu \mathrm{l}$ of $1 \times$ Apollo staining liquid was applied to each well, and incubated for $30 \mathrm{~min}$. PBS buffer containing 0.5\% TritonX100 in a volume of $100 \mu \mathrm{l}$ was used for decolorization for $10 \mathrm{~min}$. Finally, DNA staining was performed using $1 \times$ Hoechst33342 according to manufacturer's instruction. The photo was taken using florescent microscope.

\section{Migration and invasion assay by Transwell chamber}

Cell migration and invasion were investigated by Transwell chamber without or with Matrigel (BD Biosciences, San Diego, CA, USA) according to previous report [37]. Briefly, EC9706, TE1 and KYSE180 cells $\left(1 \times 10^{5}\right)$ were placed in the upper layer of chamber, whereas $20 \%$ FBS was added to underlayer of chamber. Subsequently, migratory and invasive cells were fixed using methanol, followed by staining with crystal violet $48 \mathrm{~h}$ after 
transfection. Finally, the number of invasive cells was investigated under the field of $200 \times$ magnification.

\section{Glucose uptake and lactate production assays}

Glucose and lactate assay kits (Nanjing Jiancheng Bioengineering Institute, Nanjing, China) were used to determine the glucose consumption and lactate production according to manufacturer's instructions. All data obtained were normalized to protein quantitative values.

\section{Subcellular fractionation}

Cell nucleus and cytoplasm RNA isolation kit (Beibei, Biotech, Co. Ltd., China) was used to extract the nuclear RNA and cytoplasmic RNA, respectively, according to manufacturer's instruction, and then were investigated using qRT-PCR (Supplementary Table 3).

\section{Fluorescence in situ hybridization (FISH)}

SLC2A1-AS1 probe (5'-AAAAGCAAGGCTTGGCTC ACAA-3') was synthesized and labeled using Cy3 by GenePharma Company, Shanghai, China. For FISH assay, EC9706, TE1 and KYSE180 cells were grown in 24-well plates with glass cover slips for $24 \mathrm{~h}$. After immobilization and permeabilization, EC9706, Te ard KYSE180 cells were hybridized with $20 \mu \mathrm{M} r$ y3-labe SLC2A1-AS1 probe, and 6-diamidino-2 phe vlindole (DAPI) was used to stain cell nuclei of 1 icC cer The images were observed with a florescer microscope.

\section{qRT-PCR}

Total RNA was isolated by $\operatorname{Tr} \mathrm{Ol}$ ent (Invitrogen) according to the manufact- rer's i structions. For mRNA analysis, qRT-PCR wa per ormes using Power SYBR green PCR master $\mathrm{i} x$ (. iplieu Biosystems) on an ABI 7500 series PCR achine 5 plied Biosystems using the specific primei ( $S$, plementary Table 3 ). For miR378a-3p ey pression as ay, total RNA was reverse transcribed as o the miScript Reverse Transcription Kit (Qiag Vale ir, CA). qRT-PCR amplification for miR$37-3 p$ was performed using the miScript PCR Kit (Qiag ) using the specific primers (Supplementary Table 3, Experiments were normalized to U6.

\section{Western blot}

Total proteins were extracted from ESCC cells using RIPA lysis (Solarbio, Beijing, China) and the concentration of the proteins was measured by Bradford method. The proteins were separated by SDS-PAGE, and then transferred to PVDF membranes (Millipore Corporation, Bedford, MA, USA). The primary antibodies against Ecadherin, N-cadherin, Vimentin, Glut1, HK2, PFKM, PKM, LDHA and $\beta$-actin (1: 200 dilution, Abcam, Cambridge, MA, USA) were incubated with PVDF membrane (Roche, Switzerland) overnight at room temperature after blocking with skimmed milk. Subsequently, the secondary antibody (ZSGB-BIO, Guangzhou, China) was added to PVDF membrane. Finally, enhanced chemiluminescence (ECL) reagent (Beyotime, Haime 1, China) was utilized to develop the protein signal.

\section{The detection of luciferase activity}

The dual luciferase reporter assay sys $>\mathrm{m}$ cor aucted to determine the direct interaction $\mathrm{c}$ SLC2A1-, 1 and miR378a-3p as well as miR-378a-3p an Glut1. I ecombinant vector pmirGLO-SLC2A1-AS1 SLC2A1-AS1-mutation ( $10 \mathrm{~T}$ ) a well as pmirGLO-Glut1WT and pmirGLC G 1-MUT (TSINGKE Biological Technology, Beijing China, s sequences of WT and MUT attached in Sup lem htary Table 4) along with miR-378a-3p mimic and NC and KYSE180 cells, pectively. For luciferase assay, pGL3basic with $\supset_{L}$ 1-AS1 promoter MUT and WT sequences (Suppleme ary Table 5) combined with pcDNA3.1 or p DNA3.1- 413 were transfected into EC9706, TE1 and KY, 80 cells. The Luciferase activity was determined using the Li ciferase Reporter Assay System (Promega, USA) $48 \mathrm{~h}$ a. rransfection according to manufacturer's instruction.

\section{RNA immunoprecipitation (RIP)}

RIP assay was performed in EC9706, TE1 and KYSE180 cells by using RNA-binding protein immunoprecipitation kit (Millipore company, Billerica, MA, USA) as described previously $[38,39]$. Briefly, RIP lysates were prepared from EC9706, TE1 and KYSE180 cells transfected with miR-378a-3p mimic or NC-mimic, and then were subjected to immunoprecipitation using $5 \mu \mathrm{l}$ of either a normal mouse IgG or $5 \mu \mathrm{l}$ of Anti-Ago 2 antibody and the Mana RIP ${ }^{\mathrm{rm}}$ RNA-binding Protein Immunoprecipitation Kit. The mRNA levels of SLC2A1-AS1 and miR-378a-3p enriched on beads was determined by qRT-PCR (Supplementary Table 3).

\section{In vivo nude mouse model}

Four- to six-week old female BALB/c nude mice purchased from Beijing Vital River Laboratory Animal Technology Co.,Ltd. were utilized for the animal experiment. The protocols of animal experiments were approved by the Animal Experiment Administration Committee of Zhengzhou University. For tumorigenesis assay, EC9706 cells $\left(1 \times 10^{6}\right.$ cells/each mouse $)$ were subcutaneously injected into the right flank of nude mice at their back, with 5 mice per group. When tumor volume reached around $100 \mathrm{~mm}^{3}$, NC, SLC2A1-AS1 siRNA, pcDNA3.1 and pcDNA3.1-SLC2A1-AS1 were used to inject intratumorly into the nude mice (NC and SLC2A1-AS1 siRNA modified by methylation and cholesterol, $2 \mathrm{nM}$ for each mouse; pcDNA3.1 and pcDNA3.1-SLC2A1-AS1 for each mouse with $2.5 \mu \mathrm{g}$ ) twice every week for a total of 4 weeks. 
Tumor volume was measured twice every week, and tumor growth curve was made using the formula "length $\times$ width $^{2} \times 3.14 / 6$ ". The mice were euthanized when the experiment was terminated. The tumor tissues were obtained for the isolation of total RNAs and proteins, qRT-PCR was used to investigate the SLC2A1-AS1 and miR-378a-3p expression, and Western blot was performed to determine the expressions of Glut1, HK2, PFKM, PKM and LDHA proteins.

\section{Statistical analysis}

All experimental data from at least three independent repeats were investigated using GraphPad Prism 6.0 software. Data were presented as mean with standard deviation (SD). The IHC results were examined using chisquare, and survival assay were performed using Logrank test. For the matched samples, the data was analyzed using Wilcoxon signed rank, and for non-matched samples, the data were compared by Mann-Whitney test. The comparison between two groups was determined using a Student's t-test, and the comparison of $>$ 3groups was determined using one-way ANOVA, and then Bonferroni test was selected for further statistical assay hen datasets contain $>3$ groups. A $P$ value less than 0.0 . regarded to be statistical significance.

\section{Results}

SLC2A1-AS1 is upregulated in ESCC an correlated with TNM stage, lymph node metastasis anc vatien+ ,' prognosis

In this study, we investigated SL 25,31 expression in ESCA tissues by TCGA dabase and GEO dataset, the data from TCGA dat ase revea d that SLC2A1-AS1 expression in 162 c es ESor samples was markedly higher than that 11 cases 1 normal samples (Fig. 1A), with no difference tween EAC samples and normal samples $(P>0.05)$ (Fig $1 \mathrm{~B})$, but significance difference exhibitea ${ }_{S} S C$ samples and normal samples $(P<$ $0.000^{1-}$ (Fig. $C$ ), suggesting SLC2A1-AS1 may be a $\mathrm{nc} 1 \mathrm{~b}$ omarker for discriminating EAC and ESCC histo rical types. Further GEO dataset assay demonstrated at SLC2A1-AS1 level in 7 cases of ESCC tissues was significantly higher than that in paired normal esophageal tissues (Fig. 1D). To validate these data, we detected SLC2A1-AS1 expression in 60 cases of ESCC tissues and corresponding normal tissues by qRT-PCR using SLC2A1-AS1 specific primers. The results showed that ESCC tissues displayed higher SLC2A1-AS1 expression than normal tissues (Fig. 1E). Afterwards, FISH assay demonstrated that SLC2A1-AS1 expression in ESCC tissues was dramatically higher than that in normal tissues (Fig. 1F). Meanwhile, we also found the high expression of SLC2A1-AS1 in 4 ESCC cell lines, including EC9706, TE1, KYSE180 and KYSE450 (Fig. 1G).
These findings suggest that SLC2A1-AS1 may participate in ESCC development.

To further dissect the role of SLC2A1-AS1 in progression and metastasis of ESCC, we investigated th $\mathrm{c}$ expression of SLC2A1-AS1 in ESCC patients th iverse clinicopathological features. We found that C2 1 AS1 expression was not related to th 'patients', ender, age, smoking status, drinking, diffe ontia an $\mathrm{d}$ gree and tumor size $(P>0.05)$, however, i's expressic was tightly associated with TNM stage anc lymph hode metastasis (Fig. 1H). Notably, the su ral of ESCC patients with high SLC2A1-AS1 1evel s extremely lower than that of ESCC patien $s$ th low $s$ LC2A1-AS1 level $(P<$ 0.05) (Fig. 1I). These da indicate that SLC2A1-AS1 may be a noy $\mathrm{pi}$ dictor or metastasis and prognosis for ESCC patieı.

\section{Transcrip 10. Gactor GLI3 binds to SLC2A1-AS1}

promoter a irduces its expression in ESCC

To explor possible molecular events implicated in SLC 11-AS1 high expression in ESCC, UCSC Genome Brow er (http://genome.ucsc.edu/) was used to obtain $t_{1}$ promoter sequence of SLC2A1-AS1, hTFtarget and TASPAR online software was used to predict the binding site of transcription factors, we found that transcriptional factor GLI3 bound to three potential sites in SLC2A1-AS1 promoter region (Fig. 2A). pGL3-basic with SLC2A1-AS1-promoter-WT or -MUT vectors along with pcDNA3.1 or pcDNA3.1-GLI3 were cotransfected into ESCC cells, the results demonstrated that co-transfection of pcDNA3.1-GLI3 and pGL3SLC2A1-AS1-promoter-WT extremely enhanced the activity of luciferase in EC9706, TE1 and KYSE180 cells, whereas the other groups displayed no difference (Fig. $2 B)$. To verify the precise interaction site of GLI3 in SLC2A1-AS1 promoter region, three mutation vectors harboring different SLC2A1-AS1 promoter mutation region were constructed, these vectors combined with pcDNA3.1 or pcDNA3.1-GLI3 were co-transfected to ESCC cells, we found that the mutation of site 2 significantly reduced the activity of luciferase in EC9706, TE1 and KYSE180 cells (Fig. 2C), suggesting GLI3 mainly binds to the site 2 region of SLC2A1-AS1 promoter.

To further elucidate the expression of GLI3 in ESCC, the result of TCGA database revealed that there was no difference in GLI3 expression between ESCA samples and normal samples (Fig. 2D), in which there was no difference between EAC samples and normal samples (Supplementary Fig. 1), however, significant difference was found in ESCC samples and normal samples from TCGA database (Fig. 2E), which was further confirmed by GEO dataset in 7 cases of ESCC tissues and paired normal tissues (Fig. 2F). Further investigation showed that GLI3 expression and SLC2A1-AS1 expression 


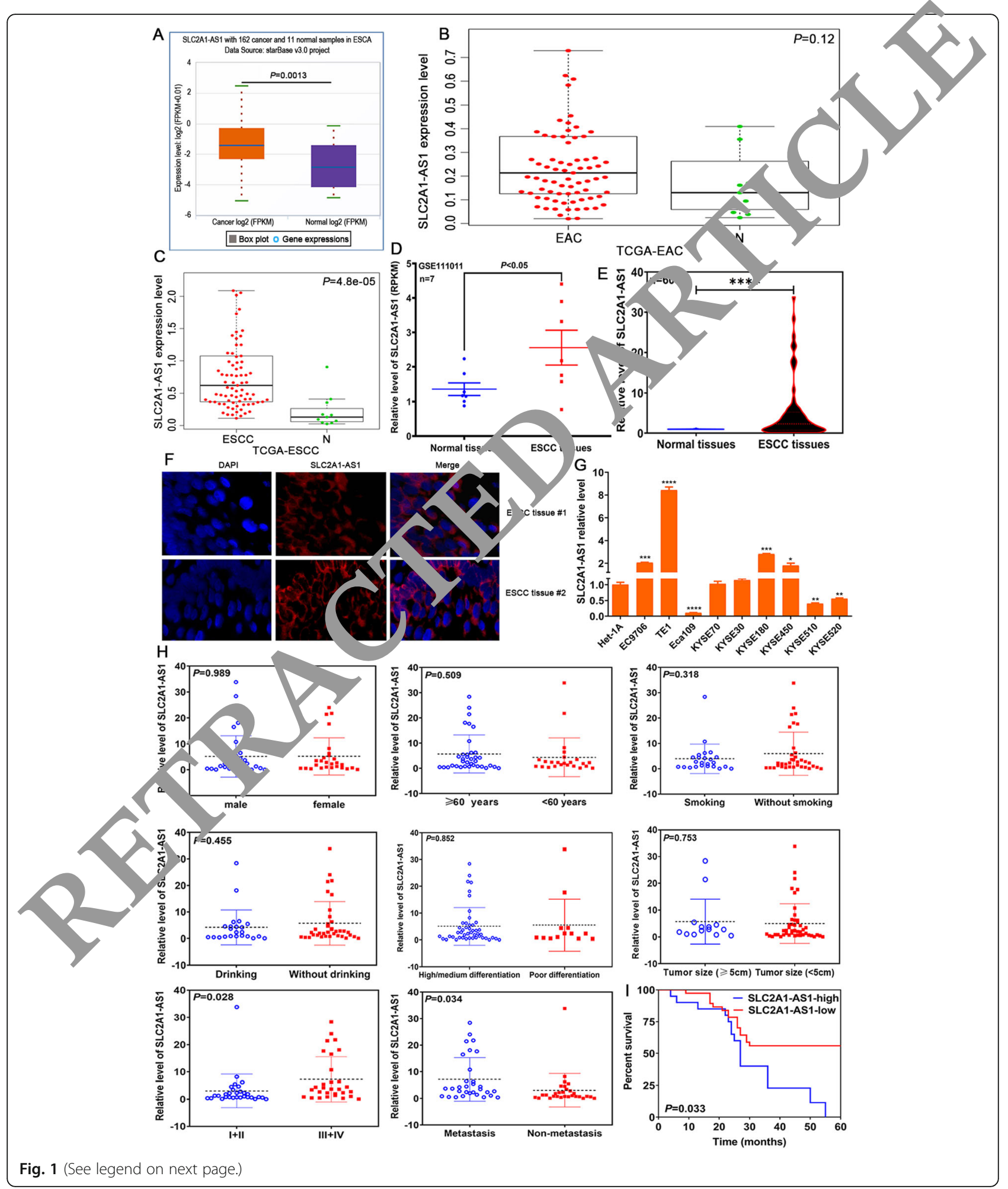


(See figure on previous page.)

Fig. 1 SLC2A1-AS1 overexpression is correlated with TNM stage, lymph node metastasis and poor prognosis in ESCC patients. A. StarBase online software assay for SLC2A1-AS1 expression level in 162 cases of ESCA tissues and 11 normal samples. B. TCGA database assay for SLC2A1-AS1 expression level in EAC samples and normal samples. C. TCGA database investigation for SLC2A1-AS1 expression level in ESCC samples na normal samples. D. GEO dataset GSE111011 analysis for SLC2A1-AS1 expression level in 7 cases of ESCC tissues and paired normal tic PCR assay for SLC2A1-AS1 expression level in 60 cases of ESCC tissues and corresponding normal tissues. F. Representative images of regarding the high expression of SLC2A1-AS1 in ESCC tissues. G. The expression of SLC2A1-AS1 was detected by qRT-PCR in a panel of EH. The correlations between SLC2A1-AS1 expression level and clinicopathological features, including gender, sex, smoking, an king, diff res riation degree, tumor size, TNM stage and lymph node metastasis. I. Log-rank test determination for the prognostic value of SI A1-A

patients. Compared to normal tissues or Het-1A cell, ${ }^{*} P<0.05,{ }^{* *} P<0.01,{ }^{* * *} P<0.001$ and ${ }^{* * * *} P<0.0001$, indicating st atistical signits nce

exhibited evidently positive correlation $(P<0.0001)$ (Fig. $2 G)$. To further verify whether GLI3 expression affected the SLC2A1-AS1 expression in ESCC cells, three GLI3 siRNAs designed were transfected to ESCC cells, we found GLI3 siRNA\#1, 2 and 3 significantly downregulated the GLI3 expression in EC9706, TE1 and KYSE180 cells (Fig. 2H), whereas pcDNA3.1-GLI3 markedly upregulated the GLI3 expression in EC9706, TE1 and KYSE180 cells (Fig. 2I). Stepwise investigation showed that GLI3 downregulation triggered the decrease of SLC2A1-AS1 expression (Fig. 2J), whereas GLI3 upregulation evoked the increase of SLC2A1-AS1 expression in EC9706, TE1 and KYSE180 cells (Fig. 2K). These andings suggest that GLI3 can bind to the promoter is $n$ of SLC2A1-AS1 and manipulate its expressio in ES cells.

Whether SLC2A1-AS1 affected GLI expres a in ESCC cells, to this end, we firstly an lyzed the possible regulatory correlation of SLC2A1-AS, by Ln ACTdb2.0 online software, we found that ${ }^{\mathrm{I}} \mathrm{C} 2 \mathrm{Al}$ - 1 might manipulate the GLI3, VEGFA, RUN XI 〈Supplementary Fig. 2A and B), furthe- inve igation showed that SLC2A1-AS1 was tigh $\mathrm{v}$ as ociate with self-sufficiency in growth signals, e dir. apopcosis, tissue invasion and metastasis as we $\mathrm{w}^{\mathrm{v}}$ s reprog imming energy metabolism (Supplementary Fig. ' C). These findings suggest that SLC2A1-A 1 is widely implicated in tumor progression, metastasis n m tabolism, therefore, we further detecter vera. 'e/ signaling pathways, which was confip ad $t$, be closely involved in tumor development and prog1 sion. We found that SLC2A1-AS1 mainly affected in and Wnt signaling pathways. SLC2A1-AS1 downregulation suppressed the expressions of GLI1, GLI3, PTCH1, CD44 and Wnt10A (Supplementary Fig. 2D), whereas SLC1A1-AS1 overexpression enhanced GLI1 and GLI3 expressions in EC9706, TE1 and KYSE180 cells (Supplementary Fig. 2E), in which GLI3 displayed the most significant alteration among all genes, suggesting that SLC2A1-AS1 manipulates GLI3 expression in feedback regulatory manner.
SLC2A1-AS1 depletion supp. ses . oliferation and induces cell apoptosis ir ESCC. "S

Sustaining proliferat of bility and resisting cell death are two representative th or hallmarks, which have been verified t, be involved in tumor development and progression. $\mathrm{H}_{0}$, urther investigated the roles of the alteration of $\mathrm{S}_{\perp} \mathrm{A} 1-\mathrm{AS} 1$ expression in cell proliferation an anoptosis in ESCC cells. Two siRNAs against $\mathrm{S}-2 \mathrm{~A} 1-\mathrm{AS} 1$ or $\mathrm{NC}$ was transfected into Fr9706, T1 1 and KYSE180 cells, qRT-PCR was used to verl the SLC2A1-AS1 expression. We found that two SLC2 1-AS1 siRNAs were both markedly downreguIa the SLC2A1-AS1 expression, in which SLC2A1As1 siRNA \#2 had the better interference effect (Fig. 3A), whereas pcDNA3.1-SLC2A1-AS1 significantly upregulated the SLC2A1-AS1 expression in EC9706, TE1 and KYSE180 cells (Supplementary Fig. 3A). Subsequently, CCK-8, colony formation and EdU staining was used to assess the proliferation ability of ESCC cells, the results demonstrated that SLC2A1-AS1 downregulation significantly suppressed cell proliferation, reduced colony formation and EdU positive staining cell numbers (Fig. 3BF), whereas the opposite data was presented after SLC2A1-AS1 overexpression (Supplementary Fig. 3B-F). Further Flow cytometry assay revealed that SLC2A1-AS1 depletion contributed to cell apoptosis (Fig. 3G and H). In contrast, SLC2A1-AS1 overexpression suppressed cell apoptosis (Supplementary Fig. 3G and H). Overall, these data imply that SLC2A1-AS1 functions as an oncogenic lncRNA in ESCC cells.

\section{SLC2A1-AS1 downregulation inhibits cell migration and invasion accompanying reduced glycolysis in ESCC cells} Metabolic reprogramming has been verified to be involved in tumor progression and metastasis through affecting the expressions of glycolysis-related proteins. Here, we found that SLC2A1-AS1 depletion suppressed cell migration and invasion in ESCC cells (Fig. 4A, B and $\mathrm{C}$ ), coupled with increased E-cadherin protein expression and reduced $\mathrm{N}$-cadherin and Vimentin protein expressions (Fig. 4D and E), whereas SLC2A1-AS1 overexpression promoted cell migration and invasion in ESCC cells (Supplementary Fig. 4 A, B and C), accompanying reduced E-cadherin protein expression and 

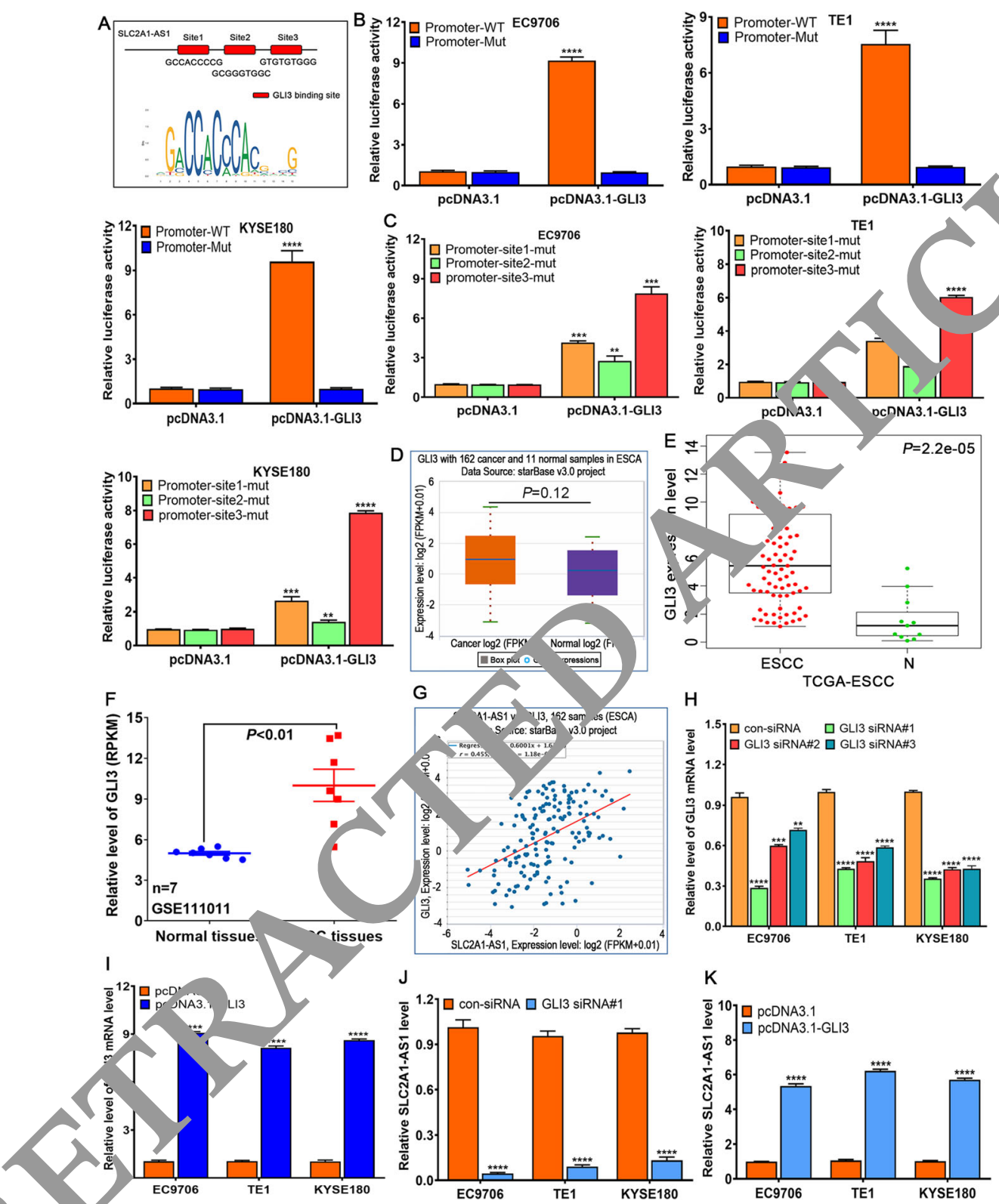

Fi 21 inscriptic, al factor GLI3 binds to the promoter region of SLC2A1-AS1 and manipulates its expression in ESCC cells. A. Three predicted GLI3 bin sim SLC2A1-AS1 promoter by hTFtarget and JASPAR online software. B and C. The luciferase reporter assay of pGL3-basic SLC2A1-AS1 promo vactor with WT or MUT GLI3 binding sites in EC9706, TE1 and KYSE180 cells transfected with pcDNA3.1 or pcDNA3.1-GLI3 vector. D. StarBase online sc. r.ware assay for GLI3 expression in ESCA tissues. E. TCGA assay for GLI3 expression level in ESCC samples and normal samples. F. GEO dataset GSE111011 assay for GLI3 expression in 7 cases of ESCC tissues and paired normal tissues. G. StarBase online software assay for the correlation between GLI3 expression and SLC2A1-AS1 expression in ESCA samples. H. The detection of GLI3 expression after transfection with GLI3 siRNAs in ESCC cells. I. GLI3 expression assay after transfection with pCDNA3.1-GLI3 vector in ESCC cells. J. GLI3 siRNA markedly downregulates the SLC2A1-AS1 expression in ESCC cells. K. GLI3 overexpression significantly upregulates the SLC2A1-AS1 level in ESCC cells. Compared to pcDNA3.1 or con-siRNA group, ${ }^{* *} P<0.01$, ${ }^{* *} P<0.001$ and ${ }^{* * *} P<0.0001$, indicating statistical significance

increased $\mathrm{N}$-cadherin and Vimentin protein expressions (Supplementary Fig. 4 D and E). Further investigation revealed that SLC2A1-AS1 depletion markedly downregulated the expression of Glut1 protein, and suppressed glucose consumption and lactate production (Fig. 4F-I), whereas SLC2A1-AS1 overexpression showed opposite effects (Supplementary Fig. 4 F-I). To further elucidate the underlying mechanisms of glucose and lactate alterations mediated by SLC2A1-AS1, GSE111011 was used to investigate the expressions of glycolysis-related proteins in 7 cases of ESCC tissues and paired normal tissues, we found that Glut1, HK2, PFKM, ALDOA, PKM, 


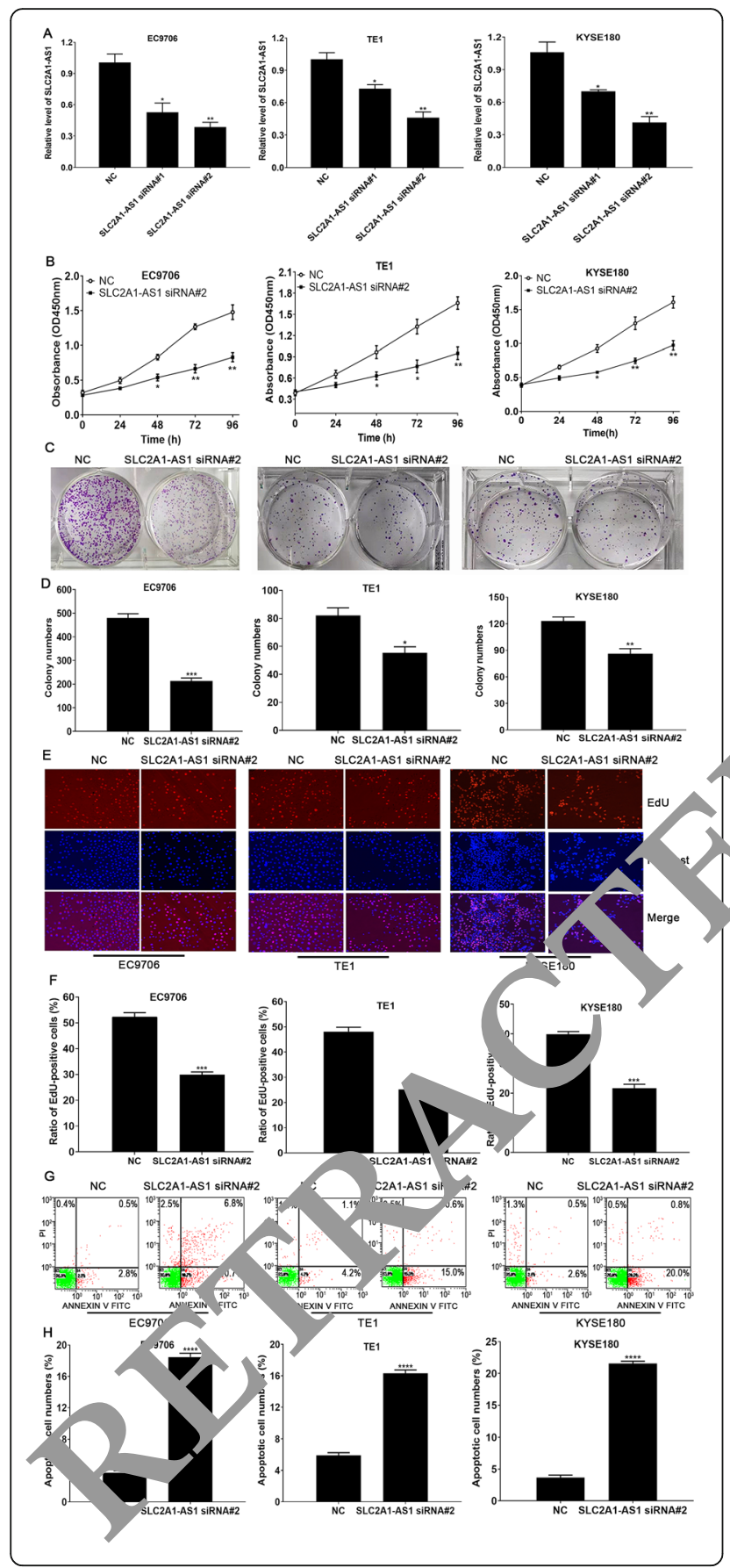

Fig. 3 SLC2A1-AS1 depletion contributes to the suppression of cell proliferation and the induction of cell apoptosis in ESCC cells. A. qRT-PCR was used to determine SLC2A1-AS1 expression following transfection with SLC2A1-AS1 siRNA in ESCC cells. B. CCK-8 assay for cell proliferation at $24 \mathrm{~h}, 48 \mathrm{~h}, 72 \mathrm{~h}$ and $96 \mathrm{~h}$ after transf tion wh SLC2A1-AS1 siRNA in ESCC cells. C. SLC2A1-AS1 downreg markedly reduced colony formation in ESCC cell $y$ D. Statistic for the number of colony formation in ESCC C. treated $\mathrm{ith}$ NC or SLC2A1-AS1 siRNA. E. EdU staining assay fo "dU-p tive $\mathrm{c}$-1 numbers in ESCC cells treated with NC $r$ SLC2A1-AS, RNA. F. Quantitative assay of the ratio of EdU- $p$ sitive cell humbers in ESCC cells treated with NC or SLC2A1-A SiR G. Fl N cytometry detection for cell apoptosis in viffe treatment ESCC cells. H. Quantitative analysis for ap totic cell mbers in a variety of ESCC cells. Compared to NC o oup $P<0.05, P<0.01,{ }^{* * *} P<0.001$ and **** $P<0.0001$, indicating statist significance

GPI, LDHA a displayed high expression in ESCC ticsues, co, ared to normal tissues (Fig. 4J), which wis w confirmed in ESCC samples, EAC samples and $n$ rma samples from TCGA database (Supplemantary $\mathrm{Fi}_{\xi}$ 5) as well as in 60 cases of ESCC samples and aired normal samples (Supplementary Fig. 6). To furth : dissect whether SLC2A1-AS1 affected the above a. ed glycolysis-related proteins, we selected 4 key proteins (HK2, PFKM, PKM and LDHA) as rate-limiting enzymes of glycolysis. We found that SLC2A1-AS1 downregulation dramatically suppressed the expressions of HK2, PFKM, PKM and LDHA proteins in EC9706, TE1 and KYSE180 cells (Fig. $4 \mathrm{~K}$ and L), whereas SLC2A1-AS1 overexpression evidently promoted the expressions of HK2, PFKM, PKM and LDHA in EC9706, TE1 and KYSE180 cells (Supplementary Fig. $4 \mathrm{~J}$ and K). These findings suggest that SLC2A1-AS1 depletion suppresses cell migration and invasion by inhibiting EMT progression and glycolysis in ESCC cells.

\section{SLC2A1-AS1 acts as a ceRNA and competitively absorbs miR-378a-3p in ESCC cells}

To investigate how SLC2A1-AS1 exerts its function in ESCC cells, qRT-PCR assay of SLC2A1-AS1 level in the nucleus and cytoplasm demonstrated that SLC2A1-AS1 was mainly localized in the cytoplasm of ESCC cells (Fig. 5A), which was further confirmed by FISH assay (Fig. 5B). Based on the localization of SLC2A1-AS1, we hypothesized that SLC2A1AS1 functioned as a ceRNA in ESCC cells, and thus DIANA LncBase Predicted v. 2 was utilized to predict the possible binding miRNAs of SLC2A1-AS1. We found that SLC2A1AS1 harbored the binding sites of miR-378a-3p (Fig. 5C). SLC2A1-AS1 expression displayed the negative correlation with miR-378a-3p in ESCA tissues (Fig. 5D). To test the interaction of SLC2A1-AS1 and miR-378a-3p in ESCC cells, Double Luciferase Report experiment was used to verify their interaction. The results demonstrated that the luciferase intensity was significantly reduced by co-transfecting miR378a-3p mimic and SLC2A1-AS1 WT, but not in the 


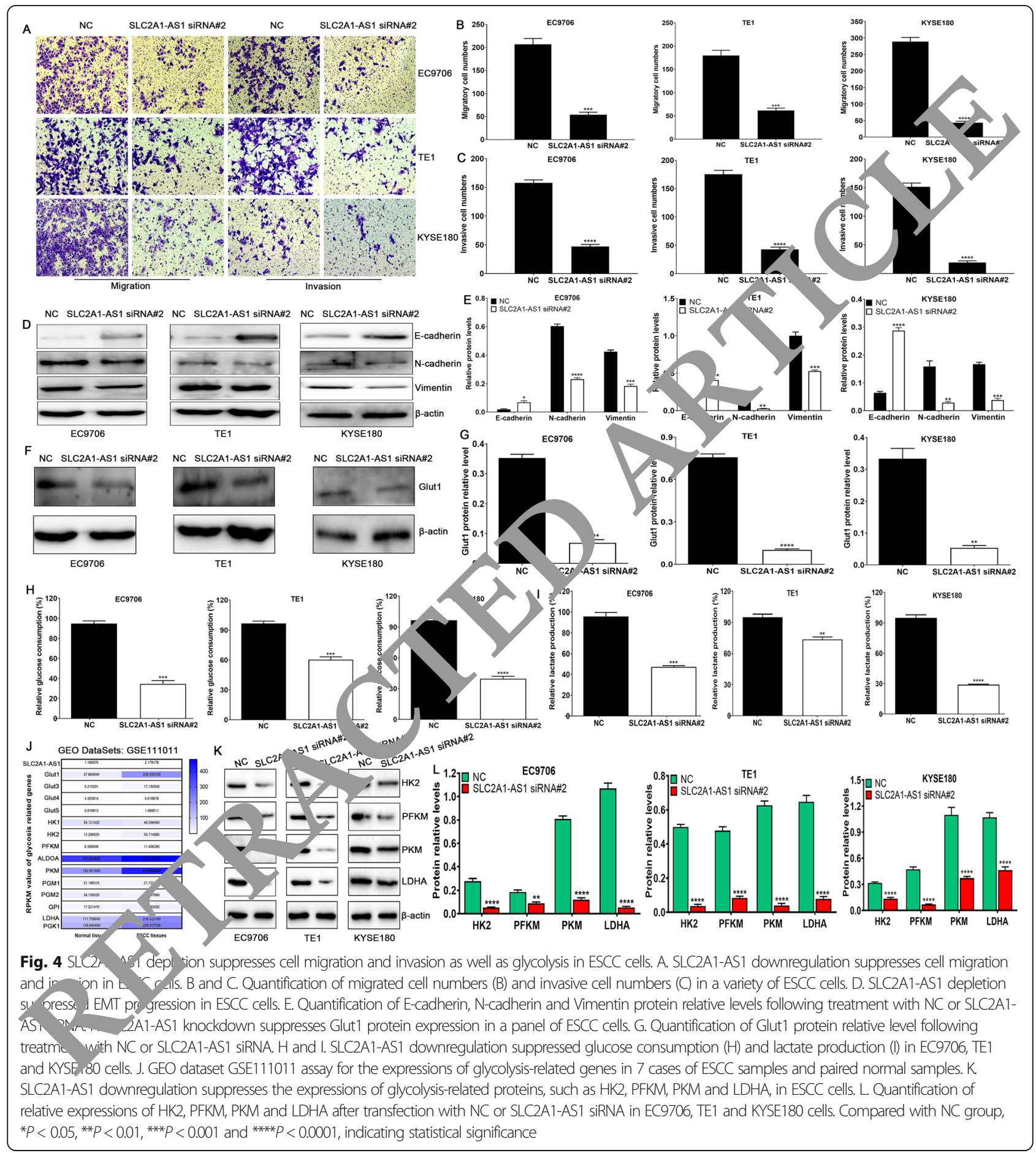

mutant vector without miR-378a-3p binding site in EC9706, TE1 and KYSE180 cells (Fig. 5E). As a core component of the RNA-induced silencing complex (RISC), Ago2 is involved in miRNA-mediating mRNA destabilization or translational repression, therefore, we further performed RIP assay by anti-Ago 2 antibody, we found that SLC2A1-AS1 and miR-378a-3p levels precipitated by anti-Ago2 antibody were dramatically increased compared to IgG group (Fig. 5F and
G). Besides, Ago2-RIP assay showed that SLC2A1-AS1 enrichment in miR-378a-3p mimic group was markedly higher than that in NC group (Fig. 5H). Subsequently, we found that SLC2A1-AS1 silencing obviously enhanced miR-378a3p level (Fig. 5I), whereas SLC2A1-AS1 overexpression dramatically reduced miR-378a-3p level in EC9706, TE1 and KYSE180 cells (Fig. 5J). These data suggest that SLC2A1AS1 directly regulates miR-378a-3p level in ESCC cells. 


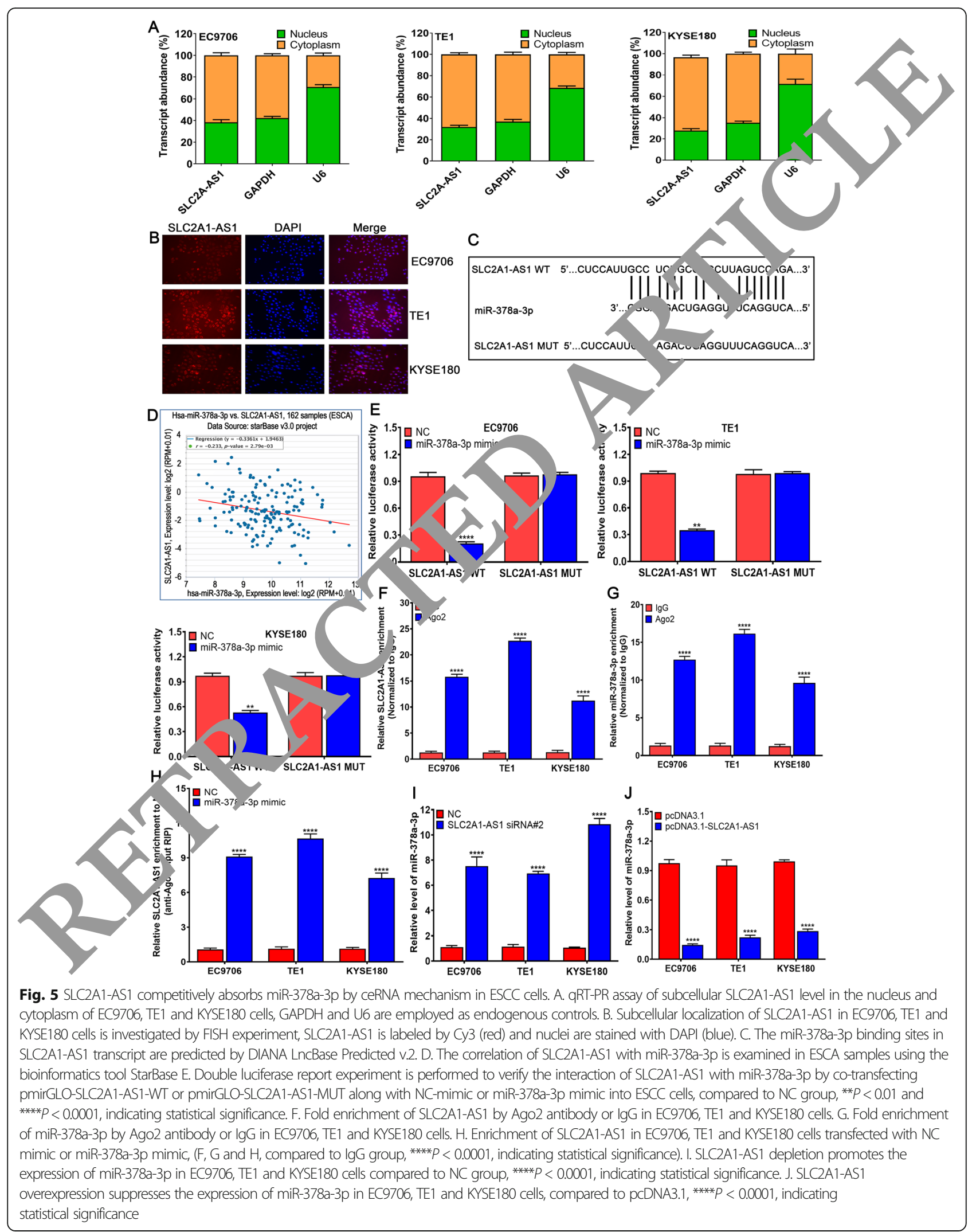




\section{The associations of miR-378a-3p and Glut1 expressions with clinicopathological features and Glut1 is a target of miR-378a-3p in ESCC cells}

To explore the possible roles of miR-378a-3p and Glut1 in ESCC development and progression, we detected their expressions and investigated their correlations with clinicopathological features. TCGA assay revealed that there was no difference in the miR-378a-3p or miR-378a expressions between ESCC and normal samples or EAC and normal samples (Fig. 6A, Supplementary Fig. 7 A and $\mathrm{B})$, but GEO assay exhibited significant difference in miR-378a-3p expression level between ESCC tissues and normal tissues (Fig. 6B), which was further validated by qRT-PCR in 60 cases of ESCC tissues and paired normal tissues (Fig. 6C) as well as ESCC cells and normal esophageal epithelial cell Het-1A (Fig. 6D). Besides, TCGA and GEO assay demonstrated that Glut1 expression in ESCC and EAC tissues was significantly higher than that in normal tissues (Fig. 6E and F, Supplementary Fig. $8 \mathrm{~A}$ and B), which was also confirmed in ESCC cells and normal esophageal epithelial cell Het-1A by Western blot (Fig. 6G and H) as well as in 60 cases of ESCC tissues and paired normal tissues by IHC ssav (Fig. 6I, Supplementary Table 6). These findings su. rfot that low miR-378a-3p or high Glut1 expressio may pa ticipate in ESCC development and progres on.

To further investigate the association $c$ miR-s $a-3 p$ and Glut1 with clinicopathological fe tures of ESCC patients, GraphPad Prism 8.0 software $n$ used to analyze the their correlations. We foun that min /8a-3p level was not related to ESCC patien s drinking and differentiation deg ee $(P>0.05)$, but was closely correlated wit tu hor s Le, TNM stage and lymph node met stas ( 1 - 0.05) (Supplementary Table 7). Beside, Glut1 e ression was not related to ESCC patients ge, 'r, age, smoking, drinking and tumor size $\quad P>0.05)$, b at was tightly correlated with differentiation gree TNM stage and lymph node metastasis 0.03 (capplementary Table 8). These findings su. est hat mar-378a-3p and Glut1 may be novel predicto for INM stage and metastasis of ESCC patients.

$\mathrm{To} \mathrm{fu}$, ner explore the regulatory role of miR-378a-3p on Glut1 in ESCC cells, miRDB online software was used to predict the target genes of miR-378a-3p. We found that Glut1 had the potential binding sites of miR378a-3p (Fig. 6J). Further Double Luciferase Report assay revealed that the luciferase intensity was significantly reduced by co-transfecting miR-378a-3p mimic and Glut1

WT, but not in the mutant vector without miR378a-3p binding site in EC9706, TE1 and KYSE180 cells (Fig. 6K). Subsequently, we detected the miR-378a-3p expression in ESCC cells by tranfecting miR-378a-3p mimic or inhibitor, we found that miR-378a-3p mimic significantly upregulated the level of miR-378a-3p, whereas miR-378a-3p inhibitor markedly downregulated the level of miR-378a-3p in EC9706, TE1 and KYSE180 cells (Fig. 6L). Western blot assay showed that miR378a-3p mimic dramatically downregulated th - expression of Glut1 protein (Fig. $6 \mathrm{M}$ and $\mathrm{N}$ ), w rea miR378a-3p inhibitor extremely upregulated the $\mathrm{e}$ res on of Glut1 protein in EC9706, TE1 a KYSE18 cells (Fig. $6 \mathrm{O}$ and P). These findings surgest hat clut1 is a direct target of miR-378a-3p in F SCC cells.

\section{The alterations of prolife acion, noptosis and invasion} triggered by miR-378 - are rev , sed by SLC2A1-AS1 and Glut1 overexpression, cSCC cells

To verify whether he biological roles of miR-378a-3p exerted in a SL, 1 - or Glut1-dependent manner, we transfected ES Cycells using NC mimic, miR-378a3p mimic 378a-3p mimic plus pcDNA3.1-SLC2A1AS1 and $r_{1} R-3 / 8 \mathrm{a}-3 \mathrm{p}$ mimic plus pcDNA3.1-Glut1 as wall as NC inhibitor, miR-378a-3p inhibitor, miR-378a$3 \mathrm{p}$ ibitor plus SLC2A1-AS1 siRNA and miR-378a-3p inhibj or plus Glut1 siRNA. We found miR-378a-3p i. $r_{A}$ c suppressed cell proliferation and invasion and increased apoptotic cell numbers (Fig. 7A-E), whereas SLC2A1-AS1 and Glut1 overexpressions recovered in part the effects in ESCC cells (Fig. 7A-E). Conversely, miR-378a-3p inhibitor promoted cell proliferation and invasion and decreased apoptotic cell numbers (Supplementary Fig. 9A-E), whereas SLC2A1-AS1 and Glut1 silencing reversed in part the effects in ESCC cells (Supplementary Fig. 9A-E). These findings revealed that miR-378a-3p functions as tumor suppressor in a SLC2A1-AS1 - or Glut1-dependent manner.

Given the links of SLC2A1-AS1 with miR-378a-3p, we put forward to whether miR-378a-3p triggered the alterations of glycolysis in ESCC and whether these changes were also reversed by SLC2A1-AS1 or Glut1. Thus, we investigated the changes of glucose consumption and lactate production as well as glycolysis-related gene expressions. We found that miR-378a-3p mimic reduced glucose consumption, lactate production and the expressions of HK2, PFKM, PKM and LDHA proteins (Fig. 7FI), whereas SLC2A1-AS1 and Glut1 overexpressions recovered in part the effects (Fig. 7F-I). Conversely, miR$378 \mathrm{a}-3 \mathrm{p}$ inhibitor promoted glucose consumption, lactate production and the expressions of HK2, PFKM, PKM and LDHA proteins (Supplementary Fig. 9F-I), whereas SLC2A1-AS1 and Glut1 silencing exhibited the opposite effects (Supplementary Fig. 9F-I). These findings suggest that SLC2A1-AS1 functions as an oncogenic lncRNA that promoted cell proliferation, migration and invasion as well as glycolysis via SLC2A1AS1/miR-378a-3p/Glut1 signal axis in ESCC cells (Fig. 7J). 


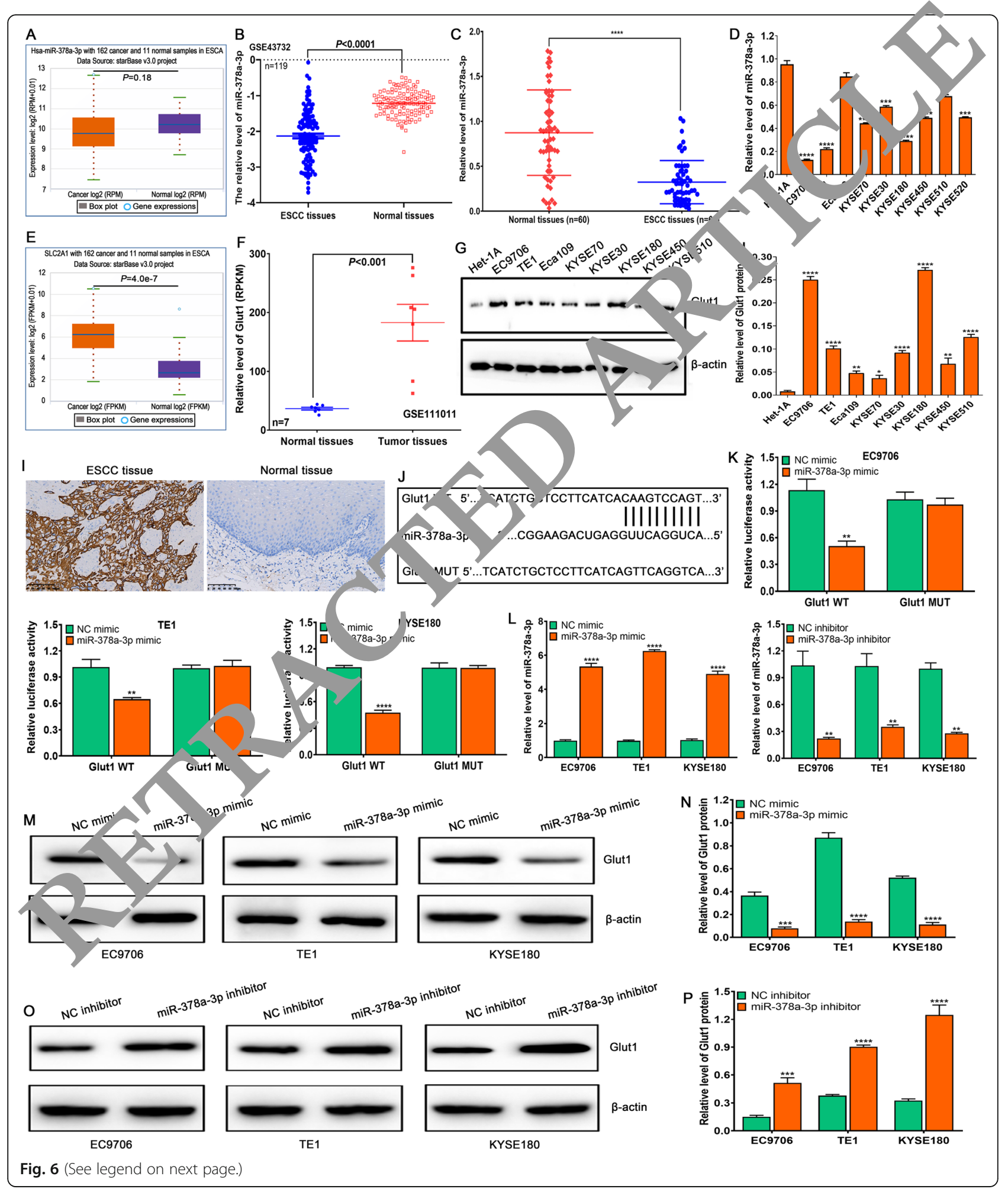


(See figure on previous page.)

Fig. 6 The expressions of miR-378a-3p and Glut1, and Glut1 is a direct target of miR-378a-3p in ESCC cells. A. The expression of miR-378a-3p is detected in 162 cases of ESCA samples and 11 cases of normal samples by StarBase online software. B. The miR-378a-3p level is investigated in 119 cases of ESCC tissues and paired normal tissues by GEO dataset GSE43732. C. The miR-378a-3p level is determined in 60 cases of ESCC tissue and paired normal tissues by qRT-PCR, compared to normal tissues, ${ }^{* * * *} P<0.0001$, indicating statistical significance. D. The expression of miR $-18 a-3$ is examined by qRT-PCR in a panel of ESCC cells, compared to Het-1 A cell, ${ }^{* *} P<0.01,{ }^{* *} P<0.001$ and ${ }^{* * *} P<0.0001$, indicating statistical sig. ance. The expression of Glut1 is detected in 162 cases of ESCA samples and 11 cases of normal samples by StarBase online software. F. The Glut1 lev investigated in 7 cases of ESCC tissues and paired normal tissues by GEO Dataset GSE111011. G. Western blot assay for Glut prou expres sonan a panel of ESCC cells and normal esophageal epithelial cell Het-1A. H. Quantification of relative levels of Glut1 in a number of prec ce. and p ormal esophageal epithelial cell Het-1A, compared to Het-1 A cell, ${ }^{*} P<0.05$, ${ }^{*} P<0.01$ and ${ }^{* * *} P<0.0001$, indicating statistical si nificance. I. I Glut1 protein expression in 60 cases of ESCC tissues and paired normal tissues. J. The miR-378a-3p binding sites in Glut1 anscript al e predicted by miRDB online software. K. Double luciferase report experiment is carried out to confirm the interaction of Glut1 wi' ' miR-1 'a-3P' y co-transfecting pmirGLO-Glut1-WT or pmirGLO-Glut1-MUT as well as NC-mimic or miR-378a-3p mimic into ESCC cells. L. miR-37-8a-3, nimic upregulates the expression of miR-378a-3p, whereas miR-378a-3p inhibitor downregulates the miR-378a-3p level in ESCC cell M. miR-3, - $-3 p$ mimic suppresses the expression of Glut1 protein in ESCC cells. N. The analysis of relative level of Glut1 protein after transfection wit YC mimic or miR-378a-3p mimic in ESCC cells. O. miR-378a-3p inhibitor enhances the expression of Glut1 protein in ESCC cells. P. The assay of relatı 'evel of Glut1 protein after transfection with NC inhibitor or miR-378a-3p inhibitor in ESCC cells. (K, L and N, compared to NC- nilr, or NC-inb, sitor, ${ }^{* *} P<0.01$, ,*** $P<0.001$ and ****P $<0.0001$, indicating statistical significance)

\section{SLC2A1-AS1 promotes tumorigenesis and glycolysis in vivo in ESCC xenografted nude mice}

To verify the roles of SLC2A1-AS1 in ESCC cells xenografted nude mice, chemically modified siRNA and overexpression vector of SLC2A1-AS1 were used to treat the tumor. The tumor growth curve demonstrated that SLC2A1-AS1 knockdown dramatically suppressecí nor growth (Fig. 8A and B), but not affected the b dy wels of nude mice (Fig. 8C), coupled with SL A1-AS downregulation and miR-378a-3p egulat $y$ in EC9706 cells xenografted tumor tissu s (Fig. 8D and E). Further Western blot assay showed at SI C2A1-AS1 depletion markedly reduced $\mathrm{t}^{\mathrm{h}}$ expresonns of Glut1, HK2, PFKM, PKM and LDHA in 706 cells xenografted tumor tissues (Fio $\mathrm{QF}$ anc G); whereas SLC2A1AS1 overexpression $d$ play ed th opposite effects on tumor growth (Fig. $\mathrm{H}$ a 1 ), mice weight (Fig. 8J), the expressions of $\mathrm{S}^{-}$2A1-AS and miR-378a-3p (Fig. 8K and L) as weli as $\mathrm{L}$ expressions of glycolysis-related proteins (Gut1 HK2, $F$ KM, PKM and LDHA) (Fig. 8M and $\mathrm{N}$ ) in $\mathrm{C} / 06$ ells xenografted tumor tissues. These data licate hat SLC2A1-AS1 contributes to ESCC to rig nesis and glycolysis in vivo.

\section{Discuss on}

Currently, a great number of lncRNAs implicated in tumor progression and glycolysis are rapidly expanding $[30,40]$. In this study, we reported a novel IncRNA SLC2A1-AS1 involved in ESCC glycolysis. SLC2A1-AS1 was frequently overexpressed in ESCC tissues and cells, and its overexpression was tightly correlated with TNM stage, lymph node metastasis and poor prognosis of ESCC patients. GLI3 as a transcriptional factor drove the SLC2A1-AS1 expression in ESCC cells, whereas SLC2A1-AS also manipulated the GLI3 expression, which formed an important regulatory feedback loop in ESCC. SLC2A1-AS1 silencing suppressed cell growth in vitro a nu vivo, migration and invasion ability, and induced $\mathrm{ce}$ ' apcptosis, coupled with alterations of EMTrolated mo cules and glycolysis-related proteins. Import tly, SLC2A1-AS1 sponged miR-378a-3p to inreas the Glut1 expression, further promoted the e. essions of glycolysis-related proteins, which led to ESCC progression and increased glycolysis, and thus targeting SLC2A1-AS1/miR-378a-3p/Glut1 signal axis may be a novel therapeutic target for ESCC patients.

Genome-wide analysis has identified a large number of differential lncRNAs in a number of tumor types. Our previous report has unveiled many differential lncRNAs in ESCA [35]. Based on this study, we focused on SLC2A1-AS1 as a novel lncRNA molecule in ESCA. We found SLC2A1-AS1 was highly expressed in ESCC tissues and cells, and its overexpression was tightly correlated with TNM stage, lymph node metastasis and poor prognosis of ESCC patients, which was similar to the study reported in LUAD [33], but was inconsistent with the data previously reported in HCC [34]. These different expression of SLC2A1-AS1 in different tumor types suggest that SLC2A1-AS1 expression may be tumor dependent. Many studies have revealed that transcriptional factors play important regulatory roles in the expressions of many genes. Wang $\mathrm{C}$, et al. found that cmyc bound to the promoter region of PVT1 to enhance the expression of PVT1 in cervical cancer [41]. HIF-1 was identified to directly bind to the promoter region of PDIA3P1, further resulting in its transcription activation in glioma [42]. Notably, the transcription factor activating enhancer binding protein $2 \alpha$ (TFAP2A) regulated the transcription of SLC2A1-AS1 by directly binding to its promoter region in LUAD cells [33]. To explore the possible regulatory factors of SLC2A1-AS1 in ESCC cells, hTFtarget and JASPAR online software were used to predict the binding sites of transcription factors in the promoter region of SLC2A1-AS1, we found that GLI3 


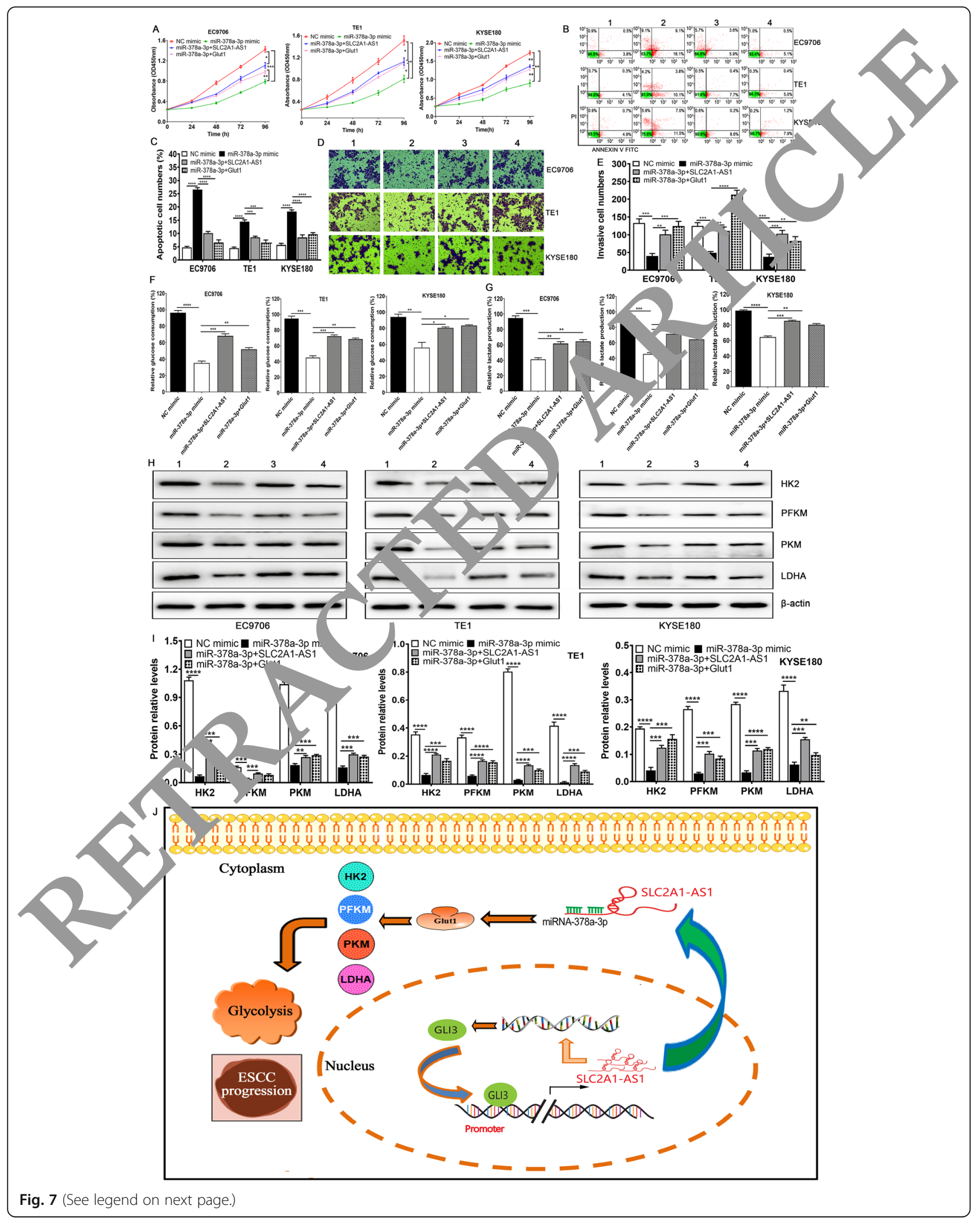


(See figure on previous page.)

Fig. 7 miR-378a-3p mimic mediated biological effects is dependent on the SLC2A1-AS1 and Glut1 expression in ESCC cells. A. ESCC cell proliferation was assessed by CCK-8 assay in NC mimic group, miR-378a-3p mimic group, miR-378a-3p plus pcDNA3.1-SLC2A1-AS1 group and miR-378a-3p plus pcDNA3.1-Glut1 group. B. Cell apoptosis was determined by Flow cytometry in the same groups appeared in cell proliferation. C. Quantitative assay of apoptotic cell unmbers in different treatment ESCC cells. D. Cell invasion ability was detected using Transwell chamber in various ESCC cells with different groups. E. Quantif ation $r f$ invasive cell numbers in diverse ESCC cells with various groups. F and G. The determination of glucose consumption (F) and lactate production (G) groups. H. Western blot assay for HK2, PFKM, PKM and LDHA protein expressions in diverse groups. I. Quantification of the relative levels of HK2, PFKM, MA and LDHA proteins in different treatment groups. ${ }^{*} P<0.05,{ }^{*} P<0.01$, ${ }^{* *} P<0.001$ and ${ }^{* * *} P<0.0001$ were regarded as statistical significan 2 A propo ${ }^{*}$ ed scheme of the roles and molecular mechanisms of SLC2A1-AS1 in ESCC progression and glycolysis is presented. Note: 1: NC mimic miR-s $-3 p$ p imic; 3 : miR-378a-3p plus SLC2A1-AS1; 4: miR-378a-3p plus Glut1

potentially bound to three sites of SLC2A1-AS1 promoter region. At present, GLI3 has been reported to be tightly associated with tumor development and progression [43, 44]. Further investigation revealed that GLI3 bound to the region of SLC2A1-AS1 promoter and promoted its expression in ESCC cells, whereas SLC2A1AS1 also affected the expression of GLI3, which formed an important regulatory feedback loop in ESCC cells, however, how SLC2A1-AS1 recruited other transcription factors affecting GLI3 expression needs to be further explored.

Increasing evidence has demonstrated that $\ln \mathrm{P}_{\mathrm{NA}} \mathrm{NA}$ are implicated in the regulation of biological proo $\mathrm{s}$, such as cell proliferation, apoptosis, invasion nd met. tasis in a multiple of tumors $[45,46]$, and na. vulation of its expression may be a novel the [47]. In this study, we verified that S C2A1-AS1 downregulation suppressed tumor grow and glycolysis in vitro and in vivo, decreasec migration and invasion and enhanced cell apoptosis, co lp th increased Ecadherin expression and reduc d expressions of $\mathrm{N}$ cadherin, Vimentin, $\mathrm{H}^{2}, \mathrm{P}, \mathrm{KM}, \mathrm{KM}$ and LDHA proteins, and conver a wure obtained following SLC2A1-AS1 ov xpressio. The alterations of these fundamental preno, es evoked by SLC2A1-AS1 suggest that LCPA1-AS may function as oncogene in ESCC and an ssential role in ESCC glycolysis.

In of eral, - cear lncRNAs play the important regulat $\mathrm{v} r$ les in chromatin structure and gene transcription Q, 4Y, whereas cytoplasmic IncRNAs function as ceRNA $\sigma$ control gene expression [50], implying that the function of lncRNAs is dependent on its subcellular localization. Given the complexity and diversity of IncRNA functions in tumor cells, we firstly examined subcellular localization of SLC2A1-AS1 in ESCC cells. We found that SLC2A1-AS1 mainly localized in cytoplasm of ESCC cells by qRT-PCR and FISH. We hypothesized that SLC2A1-AS1 exerted its function via ceRNA mechanism. Our data supported the hypothesis that SLC2A1-AS1 sponged miR-378a-3p in ESCC cells by Double Luciferase Report Assay. Stepwise investigation from Ago2-RIP assay demonstrated that Anti-Ago2 antibody markedly enriched the SLC2A1-AS1 and miR- 378a-3p in ESCC cells 10tabl when miR-378a-3p was overexpressed, the e rl ment o SLC2A1-AS1 was dramatically enhanced, sugg ting that SLC2A1-AS1 and miR-378a-3p a pec ed in the same RISC in ESCC cells. Meanwhile, SLC depletion significantly upregulated the miR-378, a level, whereas SLC2A1-AS1 overexpressio i momely downregulated the miR-378a-3p level in $\mathrm{SCC}$ cells. These findings indicate that SI C2A1-A functions as the sponge of miR-378a-3p to regu te miR-378a-3p expression in ESCC cells.

The is growing evidence that miRNAs are widely a gulated in a number of tumors, highlighting its key regulatory role in onset, growth and metastasis of tumors [51]. Emerging data suggest that miRNAs play essential regulatory roles in different physiological and pathological processes by targeting a majority of mRNAs [52]. At present, miR-378a-3p has been reported to be involved in tumor development, progression and drug resistance by manipulating a series of targeted genes. For example, miR-378 functions as an enhancer of cell growth, cell survival and angiogenesis by targeting $\mathrm{SuFu}$ and Fus-1 [53]. MiR-378a-3p has been confirmed to sensitize tumor cells to cisplatin in ovarian cancer by targeting MAPK2/GRB2 [54]. To further unveil the functions of miR-378a-3p in ESCC, we firstly examined the expression of miR-378a-3p in ESCC. We found that miR-378a-3p was frequently downregulated in ESCC tissues and cells by TCGA database, GEO dataset and qRT-PCR, and its expression was tightly associated with tumor size, TNM stage and lymph node metastasis in ESCC patients, suggesting its implication in ESCC development and progression. To uncover its underlying target genes, miRDB database was used to predict the possible downstream target genes. We found that Glut1 was a potential target of miR-378a-3p. To test the prediction, Double Luciferase Report experiment was used to validate it. We confirmed that miR-378a-3p bound to 3 '-UTR region of Glut1 to manipulate its expression in ESCC cells. MiR-378a-3p overexpression markedly suppressed the Glut1 expression, whereas miR-378a-3p inhibitor dramatically promoted the Glut1 expression in a panel of ESCC cells. These findings highlight the link of miR-378a-3p with Glut1 in ESCC cells. 


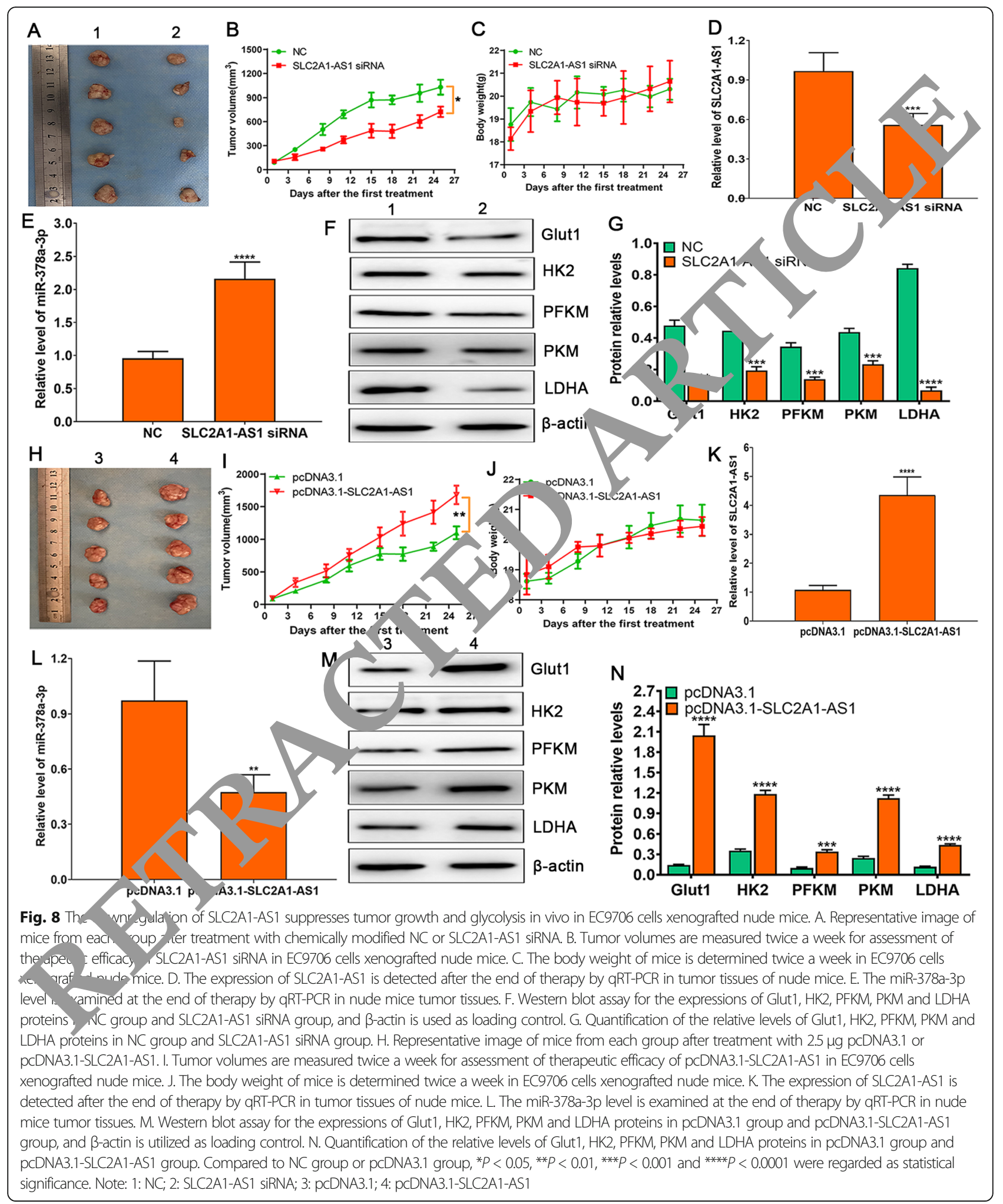

Recent investigation revealed that miR-378a-3p was recognized as the pivotal regulator of energy and glucose homeostasis [55], which will enable us to firmly believe that SLC2A1-AS1/miR-378a-3p axis participates in tumor metabolism. Wang Y, et al. verified that Lncp23145 bound to the promoter of miR-378a-3p and suppressed the expression of miR-378a-3p, further released the Glut1 expression in oral squamous cell carcinoma, 
and thus resulting in the acceleration of tumor glycolysis and tumor progression [56]. MiR-378a-3p evoked the metabolic shift by regulating PGC-1 $1 \beta / E R R \gamma$ pathway in breast carcinoma [57]. Here, we found miR-378a-3p mimic suppressed cell proliferation and invasion, promoted cell apoptosis, and triggered the decreases of glucose consumption, lactate production as well as glycolysis-related proteins (HK2, PFKM, PKM and LDHA), which was reversed in part by SLC2A1-AS1 and Glut1 overexpression, whereas miR-378a-3p inhibitor exhibited the opposite effects, which was also recovered partly by SLC2A1-AS1 and Glut1 knockdown. Overall, these findings indicate that SLC2A1-AS1/miR-378a-3p/ Glut1 may be a key player in ESCC progression and glycolysis.

\section{Conclusions}

In conclusion, SLC2A1-AS1 functions as oncogene in ESCC, and its expression is tightly associated with TNM stage, lymph node metastasis and poor prognosis. Functional and mechanistic assay suggests that SLC2A1-AS1 silencing suppresses cell growth in vitro and in vivo, migration and invasion, and expressions of glyce ysisrelated proteins by acting as a ceRNA that sponoes ir$378 \mathrm{a}-3 \mathrm{p}$, which will lead to increased Glut1 expressh and enhanced glycolysis-related protein ex ress ns. Our current results indicate that SLC2A1- 20 may $p$ y an essential role in driving tumorigenes , progression and glycolysis, and SLC2A1-AS1/miR-37 3p/G at1 signal axis may be a novel thera tic targel for ESCC patients.

\section{Abbreviations

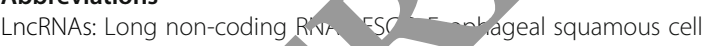 carcinoma; ESCA: Esophac can canc FAC: Esophageal adenocarcinoma; HIF- 1a: Hypoxia inducible f $\quad r-1 a$; NF-Kb Muclear factor kappa-B; Glut1: Glucose transporter 1; HK2: H rokin 2; PKM2: Pyruvate kinase type M2; LDHA: Lactate de yrógenaso eRNAs: Competing endogenous RNAs; qRT-PCR: Quar itativ real-time PCR; ENCORI: The Encyclopedia of RNA Interactomes; $k-\infty$. Cell punting Kit-8; FISH: Fluorescence in situ hybridization; ECL vhar ed chemiluminescence; RIP: RNA imm nop initatio UAD: Lung adenocarcinoma; HCC: Hepatocellular car ma -T^T2. signal transducer and activator of transcription 3; TFAP2 ranscription factor activating enhancer binding protein 2 a \\ Supplementary Information}

The online version contains supplementary material available at https://doi. org/10.1186/s13046-021-02081-8.

\section{Additional file 1. Supplementary Tables 1-8.}

Additional file 2. Supplementary Figs. 1-9.

\section{Acknowledgements}

None.

\section{Authors' contributions}

Hongtao Liu, Tianli Fan, Fangxia Guan, and Shenglei Li supervised the whole project. Hongtao Liu, Fangxia Guan and Qing Zhang designed the study. Hongtao Liu, Qing Zhang and Tianli Fan performed the majority of the experiments; Yinsen Song, Yibin Hao, Yunxia Cui and Xin Zhang contributed to animal experiments; Xueying Zhang, Yue Qin and Guangzhao Zhu performed the glucose consumption and lactate production assay; Feng Wang, Jinghan Dang, Shanshan Ma, Yanting Zhang and Wenna Guo participated in the design and interpretation of some experime ns; shenglei Li critically reviewed the paper; Fangxia Guan and Tianli Fan terpre od all results and Hongtao Liu wrote the manuscript. The author(s) ro and approved the final manuscript.

\section{Funding}

This study was supported by the National Na ural science dation of China (82073084, 81372677 and 81272691), he Key R\&D and Promotion Projects in Henan Province (No. 1821023103 ), the Na ural Science Foundation of Henan Province (No. 1 . K04 Key Scientific Research Projects of Henan Highe Educa n Institutions (No.17A180016), and the Key Discipline Constry "on Project revention and Treatment of Esophageal Cancer in Zhen zho Iniversity XKZDJC202001), the Central Plains Thousand People Plan of Her Province (204200510013), the Discipline Innovation anc isdom Int duction Plan of Higher Education in Henan Province (C 7202 ,02).

Availability of data anc "erials

The dataset. and/or analyzed during the current study are available from the corr spo riv author on reasonable request.

\section{Darlaration}

Ethics proval and consent to participate

bis sti dy was reviewed and approved by the Research and Ethics ttee of the First Affiliated Hospital of Zhengzhou University (Zhengzhou, China). The study was conducted in accordance with the international Ethical Guidelines for Biomedical Research Involving Human Subjects. All subjects provided informed consent to participate in the study.

Consent for publication

Not applicable.

\section{Competing interests}

All authors declare no competing interests.

\section{Author details}

'School of Life Sciences, Zhengzhou University, Zhengzhou 450001, Henan, China. ${ }^{2}$ Translational Medicine Research Center, Zhengzhou People's Hospital, Zhengzhou 450003, Henan, China. Institute of Genomic Medicine, College of Pharmacy, Jinan University, Guangzhou 510632, Guangdong, China. ${ }^{4}$ International Cooperative Laboratory of Traditional Chinese Medicine Modernization and Innovative Drug Development of Chinese Ministry of Education (MOE), College of pharmacy, Jinan University, Guangzhou 510632, Guangdong, China. ${ }^{5}$ Department of Clinical Medicine, Zhengzhou University, Zhengzhou 450052, Henan, China. ${ }^{6}$ Department of Pathology, the First Affiliated Hospital of Zhengzhou University, 40 Daxue Road, Zhengzhou 450052, Henan, China. ${ }^{7}$ Department of Pharmacology, School of Basic Medicine, Zhengzhou University, 100 Kexue Road, Zhengzhou 450001, Henan, China.

Received: 13 July 2021 Accepted: 20 August 2021

Published online: 13 September 2021

\section{References}

1. Roshandel G, Nourouzi A, Pourshams A, Semnani S, Merat S, Khoshnia M. Endoscopic screening for esophageal squamous cell carcinoma. Arch Iran Med. 2013;16(6):351-7.

2. Bray F, Ferlay J, Soerjomataram I, Siegel RL, Torre LA, Jemal A. Global cancer statistics 2018: GLOBOCAN estimates of incidence and mortality worldwide for 36 cancers in 185 countries. CA Cancer J Clin. 2018;68:394-424.

3. Wang C, Wang J, Chen Z, Gao Y, He J. Immunohistochemical prognostic markers of esophageal squamous cell carcinoma: a systematic review. Chin J Cancer. 2017;36(1):65. https://doi.org/10.1186/s40880-017-0232-5.

4. Fakhrian K, Ordu AD, Lordick F, Theisen J, Haller B, Omrcen T, et al. Longterm outcomes of trimodality treatment for squamous cell carcinoma of the esophagus with cisplatin and/or 5-FU: more than 20 years' experience at a 
single institution. Strahlenther Onkol. 2014;190(12):1133-40. https://doi.org/1 0.1007/s00066-014-0711-4

5. Sjoquist KM, Burmeister BH, Smithers BM, Zalcberg JR, Simes RJ, Barbour A, et al. Australasian gastro-intestinal trials G: survival after neoadjuvant chemotherapy or chemoradiotherapy for resectable oesophageal carcinoma: an updated meta-analysis. Lancet Oncol. 2011;12(7):681-92. https://doi.org/10.1016/S1470-2045(11)70142-5.

6. Shapiro J, van Lanschot JJB, Hulshof $M$, van Hagen P, van Berge Henegouwen MI, Wijnhoven BPL, et al. Neoadjuvant chemoradiotherapy plus surgery versus surgery alone for oesophageal or junctional cancer (CROSS): long-term results of a randomised controlled trial. Lancet Oncol. 2015;16(9):1090-8. https://doi.org/10.1016/S1470-2045(15)00040-6.

7. Oppedijk V, van der Gaast A, van Lanschot JJ, van Hagen P, van Os R, van Rij CM, et al. Patterns of recurrence after surgery alone versus preoperative chemoradiotherapy and surgery in the CROSS trials. J Clin Oncol. 2014;32(5): 385-91. https://doi.org/10.1200/JCO.2013.51.2186.

8. Hanahan D, Weinberg RA. Hallmarks of cancer: the next generation. Cell. 2011;144(5):646-74. https://doi.org/10.1016/j.cell.2011.02.013.

9. Warburg O, Wind F, Negelein E. The metabolism of tumors in the body. J Gen Physiol. 1927;8(6):519-30. https://doi.org/10.1085/jgp.8.6.519.

10. Racker E. Bioenergetics and the problem of tumor growth. Am Sci. 1972; 60(1):56-63.

11. Mayer A, Schmidt M, Seeger A, Serras AF, Vaupel P, Schmidberger H. GLUT-1 expression is largely unrelated to both hypoxia and the Warburg phenotype in squamous cell carcinomas of the vulva. BMC Cancer. 2014; 14(1):760. https://doi.org/10.1186/1471-2407-14-760.

12. Meng $Y, X u X$, Luan H, Li L, Dai W, Li Z, et al. The progress and development of GLUT1 inhibitors targeting cancer energy metabolism. Future Med Chem. 2019;11(17):2333-52. https://doi.org/10.4155/fmc-2019-0052.

13. Luo F, Li Y, Yuan F, Zuo J. Hexokinase II promotes the Warburg effe phosphorylating alpha subunit of pyruvate dehydrogenase. Chin 5 Res. 2019;31 (3):521-32. https://doi.org/10.21147/j.issn.1000-960 4.20 9.03.

14. Prakasam G, Singh RK, lqbal MA, Saini SK, Tiku AB, Bam ed RNK. Pyruvate kinase M knockdown-induced signaling via MP-ac ted protein kinase promotes mitochondrial biogenesi, autophagy, cancer cell survival. J Biol Chem. 2017;292(37): ( 561-76. https://doi. org/10.1074/jbc.M117.791343.

15. Wong N, Ojo D, Yan J, Tang D. PKM2 contributes ti neer retabolism. Cancer Lett. 2015;356(2):184-91. https:/ ro/10.10 lorj.canlet.2014.01.031.

16. Fantin VR, St-Pierre J, Leder P. Attenuation of 5 mpression uncovers a link between glycolysis, mitochondrial phy rogy, and tumor maintenance. Cancer Cell. 2006;9(6):425-34 /utp /doi.org 10.1016/j.ccr.2006.04.023.

17. Johnson RF, Perkins ND. N ar fa - or-kapd AB, p53, and mitochondria: regulation of cellular $m$ abolis, and tru Warburg effect. Trends Biochem Sci. 2012;37(8):317-2 https://dol. $10.1016 / j . t i b s .2012 .04 .002$.

18. Shim H, Dolde ew. C, Wu CS, Jang G, Jungmann RA, et al. c-Myc transactivation of $\mathrm{EH}-\mathrm{A}$. vlications for tumor metabolism and growth. Proc Natl Lad Sci U S A. 1) 1;94(13):6658-63. https://doi.org/10.1073/pnas. 94.13.66 -

19. Lu H, Forbes Verr A. Hypoxia-inducible factor 1 activation by aerobic Sycu is implic the Warburg effect in carcinogenesis. J Biol Chem. D02; 706):23:1 1-5. https://doi.org/10.1074/jbc.M202487200.

20. G. S, Fermundes P, O'Donovan TR, McKenna SL, Doddakula KK, Power DG, et al. volysis inhibition as a cancer treatment and its role in an antitumov, immune response. Biochim Biophys Acta. 1866;2016:87-105.

21. Akins NS, Nielson TC, Le HV. Inhibition of glycolysis and Glutaminolysis: an emerging drug discovery approach to combat Cancer. Curr Top Med Chem. 2018;18(6):494-504. https://doi.org/10.2174/1568026618666180523111351.

22. Luengo A, Gui DY, Vander Heiden MG. Targeting metabolism for Cancer therapy. Cell Chem Biol. 2017;24(9):1161-80. https://doi.org/10.1016/j. chembiol.2017.08.028.

23. Quinn JJ, Chang HY. Unique features of long non-coding RNA biogenesis and function. Nat Rev Genet. 2016;17(1):47-62. https://doi.org/10.1038/nrg.2015.10.

24. Sun $\mathrm{Q}, \mathrm{Hao} \mathrm{Q}$, Prasanth KV. Nuclear long noncoding RNAs: key regulators of gene expression. Trends Genet. 2018;34(2):142-57. https://doi.org/10.1016/j. tig.2017.11.005.

25. Ulitsky I, Bartel DP. lincRNAs: genomics, evolution, and mechanisms. Cell. 2013;154(1):26-46. https://doi.org/10.1016/j.cell.2013.06.020

26. Mondal T, Juvvuna PK, Kirkeby A, Mitra S, Kosalai ST, Traxler L, et al. Senseantisense IncRNA pair encoded by locus 6p22.3 determines neuroblastoma susceptibility via the USP36-CHD7-SOX9 regulatory Axis. Cancer Cell. 2018; 33(3):417-34 e417. https://doi.org/10.1016/j.ccell.2018.01.020.

27. Song W, Zhang J, Zhang J, Sun M, Xia Q. Overexpression of IncRNA PIK3CDAS1 promotes expression of LATS1 by competitive binding with nicroRNA566 to inhibit the growth, invasion and metastasis of hepate cellular carcinoma cells. Cancer Cell Int. 2019;19(1):150. https://do.0. 101/80/s ? 935-019-0857-3.

28. Jiang L, Zhao XH, Mao YL, Wang JF, Zheng HJ, Yoy QS. Long non aing RNA RP11-468E2.5 curtails colorectal cancer cell pro ration al 1 sumulates apoptosis via the JAK/STAT signaling pathw, targ ST, 15 and STAT6. J Exp Clin Cancer Res. 2019;38(1):4 5. https://doi.o, 10.1186/s13046019-1428-0.

29. Zhou Y, Huan L, Wu Y, Bao C, Cher B, Wà Le al IncRNA ID2-AS1 suppresses tumor metastasis by aCl. ing tho $=C 8 /$ ID2 pathway in hepatocellular carcinoma. Ca cer_Lett. D0;469:399-409. https://doi.org/1 0.1016/j.canlet.2019.11.007

30. Liao M, Liao W, Xu N, LI Liu thang S, et al. LncRNA EPB41L4A-AS1 regulates glycolysis glutamin by mediating nucleolar translocation of HDAC2. EBiol edicti . 2019;41:200-13. https://doi.org/10.1016/j.ebiom.2 019.01.035.

31. Chen H, Pei H, Hu W, J, Zhang J, Mao W, et al. Long non-coding RNA CRYBG3 regulat wycolysis of lur, cancer cells by interacting with lactate dehydrogenase a. J Cancer 20 m. 2580-8. https://doi.org/10.7150/jca.24896.

32. Malakar A Sein I, saragovi A, Winkler R, Stern-Ginossar N, Berger M, et al. Long non oding RNA MALAT1 regulates Cancer glucose metabolism by nhancing 1TOR-mediated translation of TCF7L2. Cancer Res. 2019;79(10): 2 0-93. https://doi.org/10.1158/0008-5472.CAN-18-1432.

3. Cu r, Zhang C, Ma S, Guan F. TFAP2A-induced SLC2A1-AS1 promotes chicer cell proliferation. Biol Chem. 2021;402(6):717-27. https://doi.org/10.1 15/hsz-2020-0290.

Shang R, Wang M, Dai B, Du J, Wang J, Liu Z, et al. Long noncoding RNA SLC2A1-AS1 regulates aerobic glycolysis and progression in hepatocellular carcinoma via inhibiting the STAT3/FOXM1/GLUT1 pathway. Mol Oncol. 2020;14(6):1381-96. https://doi.org/10.1002/1878-0261.12666.

35. Liu H, Zhang Q, Lou Q, Zhang X, Cui Y, Wang P, et al. Differential analysis of IncRNA, miRNA and mRNA expression profiles and the prognostic value of IncRNA in esophageal Cancer. Pathol Oncol Res. 2020;26(2):1029-39. https:// doi.org/10.1007/s12253-019-00655-8.

36. Catrina SB, Botusan IR, Rantanen A, Catrina Al, Pyakurel P, Savu O, et al. Hypoxia-inducible factor-1alpha and hypoxia-inducible factor-2alpha are expressed in kaposi sarcoma and modulated by insulin-like growth factor-l. Clin Cancer Res. 2006;12(15):4506-14. https://doi.org/10.1158/1078-0432. CCR-05-2473.

37. Zhao $Y$, Wang N, Zhang $X$, Liu H, Yang S. LnCRNA ZEB1-AS1 downregulation suppresses the proliferation and invasion by inhibiting ZEB1 expression in oesophageal squamous cell carcinoma. J Cell Mol Med. 2019; 23(12):8206-18. https://doi.org/10.1111/jcmm.14692.

38. Tsai MC, Manor O, Wan Y, Mosammaparast N, Wang JK, Lan F, et al. Long noncoding RNA as modular scaffold of histone modification complexes. Science. 2010;329(5992):689-93. https://doi.org/10.1126/science.1192002.

39. Lin C, Wang Y, Wang Y, Zhang S, Yu L, Guo C, et al. Transcriptional and posttranscriptional regulation of HOXA13 by IncRNA HOTTIP facilitates tumorigenesis and metastasis in esophageal squamous carcinoma cells. Oncogene. 2017;36(38):5392-406. https://doi.org/10.1038/onc.2017.133.

40. Chen F, Chen J, Yang L, Liu J, Zhang X, Zhang Y, et al. Extracellular vesiclepackaged HIF-1alpha-stabilizing IncRNA from tumour-associated macrophages regulates aerobic glycolysis of breast cancer cells. Nat Cell Biol. 2019;21(4):498-510. https://doi.org/10.1038/s41556-019-0299-0.

41. Wang C, Zou H, Chen A, Yang H, Yu X, Yu X, et al. C-Myc-activated long non-coding RNA PVT1 enhances the proliferation of cervical cancer cells by sponging miR-486-3p. J Biochem. 2020;167(6):565-75. https://doi.org/10.1 093/jb/mvaa005.

42. Wang S, Qi Y, Gao X, Qiu W, Liu Q, Guo X, et al. Hypoxia-induced IncRNA PDIA3P1 promotes mesenchymal transition via sponging of miR-124-3p in glioma. Cell Death Dis. 2020;11(3):168. https://doi.org/10.1038/s41419-02 0-2345-z.

43. Rodrigues M, Miguita L, De Andrade NP, Heguedusch D, Rodini CO, Moyses RA, et al. GLI3 knockdown decreases stemness, cell proliferation and invasion in oral squamous cell carcinoma. Int J Oncol. 2018;53(6):2458-72. https://doi.org/10.3892/ijo.2018.4572. 
44. Zhu H, Xia L, Shen Q, Zhao M, Gu X, Bouamar H, et al. Differential effects of GLI2 and GLI3 in regulating cervical cancer malignancy in vitro and in vivo. Lab Investig. 2018;98(11):1384-96. https://doi.org/10.1038/s41374-018-0089-5.

45. Song J, Su ZZ, Shen QM. Long non-coding RNA MALAT1 regulates proliferation, apoptosis, migration and invasion via miR-374b-5p/SRSF7 axis in non-small cell lung cancer. Eur Rev Med Pharmacol Sci. 2020;24(4):185362. https://doi.org/10.26355/eurrev_202002_20363.

46. Li WZ, Zou Y, Song ZY, Wei ZW, Chen G, Cai QL, et al. Long non-coding RNA SNHG5 affects the invasion and apoptosis of renal cell carcinoma by regulating the miR-363-3p-Twist1 interaction. Am J Transl Res. 2020;12(2): 697-707.

47. Jin SJ, Jin MZ, Xia BR, Jin WL. Long non-coding RNA DANCR as an emerging therapeutic target in human cancers. Front Oncol. 2019;9:1225. https://doi.org/10.3389/fonc.2019.01225.

48. Chaumeil J, Le Baccon P, Wutz A, Heard E. A novel role for Xist RNA in the formation of a repressive nuclear compartment into which genes are recruited when silenced. Genes Dev. 2006;20(16):2223-37. https://doi.org/1 0.1101/gad.380906.

49. Rinn JL, Kertesz M, Wang JK, Squazzo SL, Xu X, Brugmann SA, et al. Functional demarcation of active and silent chromatin domains in human HOX loci by noncoding RNAs. Cell. 2007;129(7):1311-23. https://doi.org/ 016/j.cell.2007.05.022.

50. Cesana M, Cacchiarelli D, Legnini I, Santini T, Sthandier O, Chinappin A long noncoding RNA controls muscle differentiation by fun aning a competing endogenous RNA. Cell. 2011;147(2):358-69. http. Joi.org/10.1 016/..cell.2011.09.028.

51. Lu J, Getz G, Miska EA, Alvarez-Saavedra E, Lamb J, Po D, et al. M a PNA expression profiles classify human cancers. Nature 2005;435(7043):8,4-8. https://doi.org/10.1038/nature03702.

52. Friedman RC, Farh KK, Burge CB, Bartel DP. Most in maliar mRNAs are conserved targets of microRNAs. Genom Res. 2009; is___ _-105. https:// doi.org/10.1101/gr.082701.108.

53. Lee DY, Deng Z, Wang CH, Yang BB. Micrd $D N /-3 \%$ p promotes cell survival, tumor growth, and angiogene targeti g SuFu and Fus-1 expression. Proc Natl Acad Sci U S A. 2 7; 104 1):20350 \%. https://doi.org/10.1073/pna S.0706901104.

54. Xu ZH, Yao TZ, Liu W/ mik-378a- sensitizes ovarian cancer cells to cisplatin through aly ing MAPK1) ,RB2. Biomed Pharmacother. 2018;107: 1410-7. https://a i.org/ 1016/j.biopha.2018.08.132.

55. Machado IF reodoro JS, Po círa CM, Rolo AP. miR-378a: a new emerging microRN "n me bolism. Cell Mol Life Sci. 2020;77(10):1947-58. https://doi. org/10.100, J018-0 - $03375-\mathrm{z}$.

56. W- Y, Zhan, Wang Z, Hu Q, Wu J, Li Y, et al. LncRNA-p23154 oromy es the in asion-metastasis potential of oral squamous cell carcinoma re Glut1-mediated glycolysis. Cancer Lett. 2018;434:172-83. htt, "/doj.org/10.1016/j.canlet.2018.07.016.

57. Eichne J, Perry MC, Dufour CR, Bertos N, Park M, St-Pierre J, et al. miR-378( *) mediates metabolic shift in breast cancer cells via the PGC-1beta/ ERRgamma transcriptional pathway. Cell Metab. 2010;12(4):352-61. https:// doi.org/10.1016/j.cmet.2010.09.002.

\section{Publisher's Note}

Springer Nature remains neutral with regard to jurisdictional claims in published maps and institutional affiliations.

\section{Ready to submit your research? Choose BMC and benefit from:}

- fast, convenient online submission

- thorough peer review by experienced researchers in your field

- rapid publication on acceptance

- support for research data, including large and complex data types

- gold Open Access which fosters wider collaboration and increased citations

- maximum visibility for your research: over $100 \mathrm{M}$ website views per year

At BMC, research is always in progress.

Learn more biomedcentral.com/submissions 